\title{
DIFFERENCE SCHEMES FOR NONLINEAR BVPs USING RUNGE-KUTTA IVP-SOLVERS
}

\author{
I. P. GAVRILYUK, M. HERMANN, M. V. KUTNIV, AND V. L. MAKAROV \\ Received 11 November 2005; Revised 1 March 2006; Accepted 2 March 2006
}

Difference schemes for two-point boundary value problems for systems of first-order nonlinear ordinary differential equations are considered. It was shown in former papers of the authors that starting from the two-point exact difference scheme (EDS) one can derive a so-called truncated difference scheme (TDS) which a priori possesses an arbitrary given order of accuracy $O\left(|h|^{m}\right)$ with respect to the maximal step size $|h|$. This $m$-TDS represents a system of nonlinear algebraic equations for the approximate values of the exact solution on the grid. In the present paper, new efficient methods for the implementation of an $m$-TDS are discussed. Examples are given which illustrate the theorems proved in this paper.

Copyright (c) 2006 I. P. Gavrilyuk et al. This is an open access article distributed under the Creative Commons Attribution License, which permits unrestricted use, distribution, and reproduction in any medium, provided the original work is properly cited.

\section{Introduction}

This paper deals with boundary value problems (BVPs) of the form

$$
\mathbf{u}^{\prime}(x)+A(x) \mathbf{u}=\mathbf{f}(x, \mathbf{u}), \quad x \in(0,1), \quad B_{0} \mathbf{u}(0)+B_{1} \mathbf{u}(1)=\mathbf{d},
$$

where

$$
A(x), B_{0}, B_{1}, \in \mathbb{R}^{d \times d}, \quad \operatorname{rank}\left[B_{0}, B_{1}\right]=d, \quad \mathbf{f}(x, \mathbf{u}), \mathbf{d}, \mathbf{u}(x) \in \mathbb{R}^{d},
$$

and $\mathbf{u}$ is an unknown $d$-dimensional vector-function. On an arbitrary closed irregular grid

$$
\widehat{\bar{\omega}}_{h}=\left\{x_{j}: 0=x_{0}<x_{1}<x_{2}<\cdots<x_{N}=1\right\},
$$


there exists a unique two-point exact difference scheme (EDS) such that its solution coincides with a projection of the exact solution of the BVP onto the grid $\hat{\bar{\omega}}_{h}$. Algorithmical realizations of the EDS are the so-called truncated difference schemes (TDSs). In [14] an algorithm was proposed by which for a given integer $m$ an associated TDS of the order of accuracy $m$ (or shortly $m$-TDS) can be developed.

The EDS and the corresponding three-point difference schemes of arbitrary order of accuracy $m$ (so-called truncated difference schemes of rank $m$ or shortly $m$-TDS) for BVPs for systems of second-order ordinary differential equations (ODEs) with piecewise continuous coefficients were constructed in $[8-18,20,23,24]$. These ideas were further developed in [14] where two-point EDS and TDS of an arbitrary given order of accuracy for problem (1.1) were proposed. One of the essential parts of the resulting algorithm was the computation of the fundamental matrix which influenced considerably its complexity. Another essential part was the use of a Cauchy problem solver (IVP-solver) on each subinterval $\left[x_{j-1}, x_{j}\right]$ where a one-step Taylor series method of the order $m$ has been chosen. This supposes the calculation of derivatives of the right-hand side which negatively influences the efficiency of the algorithm.

The aim of this paper is to remove these two drawbacks and, therefore, to improve the computational complexity and the effectiveness of TDS for problem (1.1). We propose a new implementation of TDS with the following main features: (1) the complexity is significantly reduced due to the fact that no fundamental matrix must be computed; (2) the user can choose an arbitrary one-step method as the IVP-solver. In our tests we have considered the Taylor series method, Runge-Kutta methods, and the fixed point iteration for the equivalent integral equation. The efficiency of 6th- and 10th-order accurate TDS is illustrated by numerical examples. The proposed algorithm can also be successfully applied to BVPs for systems of stiff ODEs without use of the "expensive" IVP-solvers.

Note that various modifications of the multiple shooting method are considered to be most efficient for problem (1.1) $[2,3,6,22]$. The ideas of these methods are very close to that of EDS and TDS and are based on the successive solution of IVPs on small subintervals. Although there exist a priori estimates for all IVP-solver in use, to our best knowledge only a posteriori estimates for the shooting method are known.

The theoretical framework of this paper allows to carry out a rigorous mathematical analysis of the proposed algorithms including existence and uniqueness results for EDS and TDS, a priori estimates for TDS (see, e.g., Theorem 4.2), and convergence results for an iterative procedure of its practical implementation.

The paper is organized as follows. In Section 2, leaning on [14], we discuss the properties of the BVP under consideration including the existence and uniqueness of solutions. Section 3 deals with the two-point exact difference schemes and a result about the existence and uniqueness of solutions. The main result of the paper is contained in Section 4 . We represent efficient algorithm for the implementation of EDS by TDS of arbitrary given order of accuracy $m$ and give its theoretical justification with a priori error estimates. Numerical examples confirming the theoretical results as well as a comparison with the multiple shooting method are given. 


\section{The given BVP: existence and uniqueness of the solution}

The linear part of the differential equation in (1.1) determines the fundamental matrix (or the evolution operator) $U(x, \xi) \in \mathbb{R}^{d \times d}$ which satisfies the matrix initial value problem (IVP)

$$
\frac{\partial U(x, \xi)}{\partial x}+A(x) U(x, \xi)=0, \quad 0 \leq \xi \leq x \leq 1, \quad U(\xi, \xi)=I,
$$

where $I \in \mathbb{R}^{d \times d}$ is the identity matrix. The fundamental matrix $U$ satisfies the semigroup property

$$
U(x, \xi) U(\xi, \eta)=U(x, \eta)
$$

and the inequality (see [14])

$$
\|U(x, \xi)\| \leq \exp \left[c_{1}(x-\xi)\right]
$$

In what follows we denote by $\|\mathbf{u}\| \equiv \sqrt{\mathbf{u}^{T} \mathbf{u}}$ the Euclidean norm of $\mathbf{u} \in \mathbb{R}^{d}$ and we will use the subordinate matrix norm generated by this vector norm. For vector-functions $\mathbf{u}(x) \in \mathbb{C}[0,1]$, we define the norm

$$
\|\mathbf{u}\|_{0, \infty,[0,1]}=\max _{x \in[0,1]}\|\mathbf{u}(x)\|
$$

Let us make the following assumptions.

(PI) The linear homogeneous problem corresponding to (1.1) possesses only the trivial solution.

(PII) For the elements of the matrix $A(x)=\left[a_{i j}(x)\right]_{i, j=1}^{d}$ it holds that $a_{i j}(x) \in \mathbb{C}[0,1]$, $i, j=1,2, \ldots, d$.

The last condition implies the existence of a constant $c_{1}$ such that

$$
\|A(x)\| \leq c_{1} \quad \forall x \in[0,1] .
$$

It is easy to show that condition (PI) guarantees the nonsingularity of the matrix $Q \equiv$ $B_{0}+B_{1} U(1,0)$ (see, e.g., [14]).

Some sufficient conditions which guarantee that the linear homogeneous BVP corresponding to (1.1) has only the trivial solution are given in [14].

Let us introduce the vector-function

$$
\mathbf{u}^{(0)}(x) \equiv U(x, 0) Q^{-1} \mathbf{d}
$$

(which exists due to assumption (PI) for all $x \in[0,1]$ ) and the set

$$
\begin{gathered}
\Omega(D, \beta(\cdot)) \equiv\left\{\mathbf{v}(x)=\left(v_{i}(x)\right)_{i=1}^{d}, v_{i}(x) \in \mathbb{C}[0,1], i=1,2, \ldots, d,\right. \\
\left.\left\|\mathbf{v}(x)-\mathbf{u}^{(0)}(x)\right\| \leq \beta(x), x \in D\right\},
\end{gathered}
$$

where $D \subseteq[0,1]$ is a closed set, and $\beta(x) \in \mathbb{C}[0,1]$. 
Further, we assume the following assumption.

(PIII) The vector-function $\mathbf{f}(x, \mathbf{u})=\left\{f_{j}(x, \mathbf{u})\right\}_{j=1}^{d}$ satisfies the conditions

$$
\begin{gathered}
f_{j}(x, \mathbf{u}) \in C([0,1] \times \Omega([0,1], r(\cdot))), \quad\|\mathbf{f}(x, \mathbf{u})\| \leq K \quad \forall x \in[0,1], \mathbf{u} \in \Omega([0,1], r(\cdot)), \\
\|\mathbf{f}(x, \mathbf{u})-\mathbf{f}(x, \mathbf{v})\| \leq L\|\mathbf{u}-\mathbf{v}\| \quad \forall x \in[0,1], \mathbf{u}, \mathbf{v} \in \Omega([0,1], r(\cdot)), \\
r(x) \equiv K \exp \left(c_{1} x\right)\left[x+\|H\| \exp \left(c_{1}\right)\right],
\end{gathered}
$$

where $H \equiv Q^{-1} B_{1}$.

Now, we discuss sufficient conditions which guarantee the existence and uniqueness of a solution of problem (1.1). We will use these conditions below to prove the existence of the exact two-point difference scheme and to justify the schemes of an arbitrary given order of accuracy.

We begin with the following statement.

Theorem 2.1. Under assumptions (PI)-(PIII) and

$$
q \equiv L \exp \left(c_{1}\right)\left[1+\|H\| \exp \left(c_{1}\right)\right]<1,
$$

problem (1.1) possesses in the set $\Omega([0,1], r(\cdot))$ a unique solution $\mathbf{u}(x)$ which can be determined by the iteration procedure

$$
\mathbf{u}^{(k)}(x)=\int_{0}^{1} G(x, \xi) \mathbf{f}\left(\xi, \mathbf{u}^{(k-1)}(\xi)\right) d \xi+\mathbf{u}^{(0)}(x), \quad x \in[0,1],
$$

with the error estimate

$$
\left\|\mathbf{u}^{(k)}-\mathbf{u}\right\|_{0, \infty,[0,1]} \leq \frac{q^{k}}{1-q} r(1)
$$

where

$$
G(x, \xi) \equiv \begin{cases}-U(x, 0) H U(1, \xi), & 0 \leq x \leq \xi \\ -U(x, 0) H U(1, \xi)+U(x, \xi), & \xi \leq x \leq 1\end{cases}
$$

\section{Existence of an exact two-point difference scheme}

Let us consider the space of vector-functions $\left(\mathbf{u}_{j}\right)_{j=0}^{N}$ defined on the grid $\hat{\bar{\omega}}_{h}$ and equipped with the norm

$$
\|\mathbf{u}\|_{0, \infty, \hat{\bar{\omega}}_{h}}=\max _{0 \leq j \leq N}\left\|\mathbf{u}_{j}\right\|
$$

Throughout the paper $M$ denotes a generic positive constant independent of $|h|$.

Given $\left(\mathbf{v}_{j}\right)_{j=0}^{N} \subset \mathbb{R}^{d}$ we define the IVPs (each of the dimension $d$ )

$$
\begin{gathered}
\frac{d \mathbf{Y}^{j}\left(x, \mathbf{v}_{j-1}\right)}{d x}+ \\
A(x) \mathbf{Y}^{j}\left(x, \mathbf{v}_{j-1}\right)=\mathbf{f}\left(x, \mathbf{Y}^{j}\left(x, \mathbf{v}_{j-1}\right)\right), \quad x \in\left(x_{j-1}, x_{j}\right] \\
\mathbf{Y}^{j}\left(x_{j-1}, \mathbf{v}_{j-1}\right)=\mathbf{v}_{j-1}, \quad j=1,2, \ldots, N
\end{gathered}
$$


The existence of a unique solution of (3.2) is postulated in the following lemma.

LEMma 3.1. Let assumptions (PI)-(PIII) be satisfied. If the grid vector-function $\left(\mathbf{v}_{j}\right)_{j=0}^{N} b e$ longs to $\Omega\left(\hat{\bar{\omega}}_{h}, r(\cdot)\right)$, then the problem (3.2) has a unique solution.

Proof. The question about the existence and uniqueness of the solution to (3.2) is equivalent to the same question for the integral equation

$$
\mathbf{Y}^{j}\left(x, \mathbf{v}_{j-1}\right)=\mathfrak{J}\left(x, \mathbf{v}_{j-1}, \mathbf{Y}^{j}\right)
$$

where

$$
\mathfrak{J}\left(x, \mathbf{v}_{j-1}, \mathbf{Y}^{j}\right) \equiv U\left(x, x_{j-1}\right) \mathbf{v}_{j-1}+\int_{x_{j-1}}^{x} U(x, \xi) \mathbf{f}\left(\xi, \mathbf{Y}^{j}\left(\xi, \mathbf{v}_{j-1}\right)\right) d \xi, \quad x \in\left[x_{j-1}, x_{j}\right] .
$$

We define the $n$th power of the operator $\mathfrak{J}\left(x, \mathbf{v}_{j-1}, \mathbf{Y}^{j}\right)$ by

$$
\mathfrak{I}^{n}\left(x, \mathbf{v}_{j-1}, \mathbf{Y}^{j}\right)=\mathfrak{I}\left(x, \mathbf{v}_{j-1}, \mathfrak{I}^{n-1}\left(x, \mathbf{v}_{j-1}, \mathbf{Y}^{j}\right)\right), \quad n=2,3, \ldots
$$

Let $\mathbf{Y}^{j}\left(x, \mathbf{v}_{j-1}\right) \in \Omega\left(\left[x_{j-1}, x_{j}\right], r(\cdot)\right)$ for $\left(\mathbf{v}_{j}\right)_{j=0}^{N} \in \Omega\left(\hat{\bar{\omega}}_{h}, r(\cdot)\right)$. Then

$$
\begin{aligned}
\left\|\mathfrak{I}\left(x, \mathbf{v}_{j-1}, \mathbf{Y}^{j}\right)-\mathbf{u}^{(0)}(x)\right\| \\
\quad \leq\left\|U\left(x, x_{j-1}\right)\right\|\left\|\mathbf{v}_{j-1}-\mathbf{u}^{(0)}\left(x_{j-1}\right)\right\|+\int_{x_{j-1}}^{x}\|U(x, \xi)\|\left\|\mathbf{f}\left(\xi, \mathbf{Y}^{j}\left(\xi, \mathbf{v}_{j-1}\right)\right)\right\| d \xi \\
\quad \leq K \exp \left(c_{1} x\right)\left[x_{j-1}+\|H\| \exp \left(c_{1}\right)\right]+K\left(x-x_{j-1}\right) \exp \left[c_{1}\left(x-x_{j-1}\right)\right] \\
\quad \leq K \exp \left(c_{1} x\right)\left[x+\|H\| \exp \left(c_{1}\right)\right]=r(x), \quad x \in\left[x_{j-1}, x_{j}\right],
\end{aligned}
$$

that is, for grid functions $\left(\mathbf{v}_{j}\right)_{j=0}^{N} \in \Omega\left(\hat{\bar{\omega}}_{h}, r(\cdot)\right)$ the operator $\mathfrak{J}\left(x, \mathbf{v}_{j-1}, \mathbf{Y}^{j}\right)$ transforms the set $\Omega\left(\left[x_{j-1}, x_{j}\right], r(\cdot)\right)$ into itself.

Besides, for $\mathbf{Y}^{j}\left(x, \mathbf{v}_{j-1}\right), \tilde{\mathbf{Y}}^{j}\left(x, \mathbf{v}_{j-1}\right) \in \Omega\left(\left[x_{j-1}, x_{j}\right], r(\cdot)\right)$, we have the estimate

$$
\begin{aligned}
& \left\|\mathfrak{I}\left(x, \mathbf{v}_{j-1}, \mathbf{Y}^{j}\right)-\mathfrak{I}\left(x, \mathbf{v}_{j-1}, \tilde{\mathbf{Y}}^{j}\right)\right\| \\
& \quad \leq \int_{x_{j-1}}^{x}\|U(x, \xi)\|\left\|\mathbf{f}\left(\xi, \mathbf{Y}^{j}\left(\xi, \mathbf{v}_{j-1}\right)\right)-\mathbf{f}\left(\xi, \tilde{\mathbf{Y}}^{j}\left(\xi, \mathbf{v}_{j-1}\right)\right)\right\| d \xi \\
& \quad \leq L \exp \left(c_{1} h_{j}\right) \int_{x_{j-1}}^{x}\left\|\mathbf{Y}^{j}\left(\xi, \mathbf{v}_{j-1}\right)-\tilde{\mathbf{Y}}^{j}\left(\xi, \mathbf{v}_{j-1}\right)\right\| d \xi \\
& \quad \leq L \exp \left(c_{1} h_{j}\right)\left(x-x_{j-1}\right)\left\|\mathbf{Y}^{j}-\tilde{\mathbf{Y}}^{j}\right\|_{0, \infty,\left[x_{j-1}, x_{j}\right]} \cdot
\end{aligned}
$$


Using this estimate, we get

$$
\begin{aligned}
& \left\|\mathfrak{J}^{2}\left(x, \mathbf{v}_{j-1}, \mathbf{Y}^{j}\right)-\mathfrak{J}^{2}\left(x, \mathbf{v}_{j-1}, \tilde{\mathbf{Y}}^{j}\right)\right\| \\
& \quad \leq L \exp \left(c_{1} h_{j}\right) \int_{x_{j-1}}^{x}\left\|\mathfrak{I}\left(x, \mathbf{v}_{j-1}, \mathbf{Y}^{j}\right)-\mathfrak{I}\left(x, \mathbf{v}_{j-1}, \tilde{\mathbf{Y}}^{j}\right)\right\| d \xi \\
& \quad \leq \frac{\left[L \exp \left(c_{1} h_{j}\right)\left(x-x_{j-1}\right)\right]^{2}}{2 !}\left\|\mathbf{Y}^{j}-\tilde{\mathbf{Y}}^{j}\right\|_{0, \infty,\left[x_{j-1}, x_{j}\right]} .
\end{aligned}
$$

If we continue to determine such estimates, we get by induction

$$
\left\|\mathfrak{I}^{n}\left(x, \mathbf{v}_{j-1}, \mathbf{Y}^{j}\right)-\mathfrak{J}^{n}\left(x, \mathbf{v}_{j-1}, \tilde{\mathbf{Y}}^{j}\right)\right\| \leq \frac{\left[L \exp \left(c_{1} h_{j}\right)\left(x-x_{j-1}\right)\right]^{n}}{n !}\left\|\mathbf{Y}^{j}-\tilde{\mathbf{Y}}^{j}\right\|_{0, \infty,\left[x_{j-1}, x_{j}\right]}
$$

and it follows that

$$
\left\|\mathfrak{I}^{n}\left(\cdot, \mathbf{v}_{j-1}, \mathbf{Y}^{j}\right)-\mathfrak{I}^{n}\left(\cdot, \mathbf{v}_{j-1}, \tilde{\mathbf{Y}}^{j}\right)\right\|_{0, \infty,\left[x_{j-1}, x_{j}\right]} \leq \frac{\left[L \exp \left(c_{1} h_{j}\right) h_{j}\right]^{n}}{n !}\left\|\mathbf{Y}^{j}-\tilde{\mathbf{Y}}^{j}\right\|_{0, \infty,\left[x_{j-1}, x_{j}\right]} \cdot
$$

Taking into account that $\left[L \exp \left(c_{1} h_{j}\right) h_{j}\right]^{n} /(n !) \rightarrow 0$ for $n \rightarrow \infty$, we can fix $n$ large enough such that $\left[\operatorname{Lexp}\left(c_{1} h_{j}\right) h_{j}\right]^{n} /(n !)<1$, which yields that the $n$th power of the operator $\mathfrak{J}^{n}\left(x, \mathbf{v}_{j-1}, \mathbf{Y}^{j}\right)$ is a contractive mapping of the set $\Omega\left(\left[x_{j-1}, x_{j}\right], r(\cdot)\right)$ into itself. Thus (see, e.g., [1] or [25]), for $\left(\mathbf{v}_{j}\right)_{j=0}^{N} \in \Omega\left(\hat{\bar{\omega}}_{h}, r(x)\right)$, problem (3.3) (or problem (3.2)) has a unique solution.

We are now in the position to prove the main result of this section.

Theorem 3.2. Let the assumptions of Theorem 2.1 be satisfied. Then, there exists a twopoint EDS for problem (1.1). It is of the form

$$
\begin{gathered}
\mathbf{u}_{j}=\mathbf{Y}^{j}\left(x_{j}, \mathbf{u}_{j-1}\right), \quad j=1,2, \ldots, N, \\
B_{0} \mathbf{u}_{0}+B_{1} \mathbf{u}_{N}=\mathbf{d} .
\end{gathered}
$$

Proof. It is easy to see that

$$
\begin{gathered}
\frac{d}{d x} \mathbf{Y}^{j}\left(x, \mathbf{u}_{j-1}\right)+ \\
A(x) \mathbf{Y}^{j}\left(x, \mathbf{u}_{j-1}\right)=\mathbf{f}\left(x, \mathbf{Y}^{j}\left(x, \mathbf{u}_{j-1}\right)\right), \quad x \in\left(x_{j-1}, x_{j}\right] \\
\mathbf{Y}^{j}\left(x_{j-1}, \mathbf{u}_{j-1}\right)=\mathbf{u}_{j-1}, \quad j=1,2, \ldots, N .
\end{gathered}
$$

Due to Lemma 3.1 the solvability of the last problem is equivalent to the solvability of problem (1.1). Thus, the solution of problem (1.1) can be represented by

$$
\mathbf{u}(x)=\mathbf{Y}^{j}\left(x, \mathbf{u}_{j-1}\right), \quad x \in\left[x_{j-1}, x_{j}\right], j=1,2, \ldots, N .
$$

Substituting here $x=x_{j}$, we get the two-point EDS (3.11)-(3.12). 
For the further investigation of the two-point EDS, we need the following lemma.

Lemma 3.3. Let the assumptions of Lemma 3.1 be satisfied. Then, for two grid functions $\left(\mathbf{u}_{j}\right)_{j=0}^{N}$ and $\left(\mathbf{v}_{j}\right)_{j=0}^{N}$ in $\Omega\left(\hat{\bar{\omega}}_{h}, r(\cdot)\right)$,

$$
\begin{aligned}
& \left\|\mathbf{Y}^{j}\left(x, \mathbf{u}_{j-1}\right)-\mathbf{Y}^{j}\left(x, \mathbf{v}_{j-1}\right)-U\left(x, x_{j-1}\right)\left(\mathbf{u}_{j-1}-\mathbf{v}_{j-1}\right)\right\| \\
& \quad \leq L\left(x-x_{j-1}\right) \exp \left\{c_{1}\left(x-x_{j-1}\right)+L \int_{x_{j-1}}^{x} \exp \left[c_{1}(x-\xi)\right] d \xi\right\}\left\|\mathbf{u}_{j-1}-\mathbf{v}_{j-1}\right\| .
\end{aligned}
$$

Proof. When proving Lemma 3.1, it was shown that $\mathbf{Y}^{j}\left(x, \mathbf{u}_{j-1}\right), \mathbf{Y}^{j}\left(x, \mathbf{v}_{j-1}\right)$ belong to $\Omega\left(\left[x_{j-1}, x_{j}\right], r(\cdot)\right)$. Therefore it follows from (3.2) that

$$
\begin{aligned}
& \left\|\mathbf{Y}^{j}\left(x, \mathbf{u}_{j-1}\right)-\mathbf{Y}^{j}\left(x, \mathbf{v}_{j-1}\right)-U\left(x, x_{j-1}\right)\left(\mathbf{u}_{j-1}-\mathbf{v}_{j-1}\right)\right\| \\
& \leq L \int_{x_{j-1}}^{x} \exp \left[c_{1}(x-\xi)\right]\left\{\exp \left[c_{1}\left(\xi-x_{j-1}\right)\right]\left\|\mathbf{u}_{j-1}-\mathbf{v}_{j-1}\right\|\right. \\
& \left.\quad+\left\|\mathbf{Y}^{j}\left(\xi, \mathbf{u}_{j-1}\right)-\mathbf{Y}^{j}\left(\xi, \mathbf{v}_{j-1}\right)-U\left(\xi, x_{j-1}\right)\left(\mathbf{u}_{j-1}-\mathbf{v}_{j-1}\right)\right\|\right\} d \xi \\
& =L \exp \left[c_{1}\left(x-x_{j-1}\right)\right]\left(x-x_{j-1}\right)\left\|\mathbf{u}_{j-1}-\mathbf{v}_{j-1}\right\| \\
& \quad+L \int_{x_{j-1}}^{x} \exp \left[c_{1}(x-\xi)\right]\left\|\mathbf{Y}^{j}\left(\xi, \mathbf{u}_{j-1}\right)-\mathbf{Y}^{j}\left(\xi, \mathbf{v}_{j-1}\right)-U\left(\xi, x_{j-1}\right)\left(\mathbf{u}_{j-1}-\mathbf{v}_{j-1}\right)\right\| d \xi
\end{aligned}
$$

Now, Gronwall's lemma implies (3.15).

We can now prove the uniqueness of the solution of the two-point EDS (3.11)-(3.12).

Theorem 3.4. Let the assumptions of Theorem 2.1 be satisfied. Then there exists an $h_{0}>0$ such that for $|h| \leq h_{0}$ the two-point EDS (3.11)-(3.12) possesses a unique solution $\left(\mathbf{u}_{j}\right)_{j=0}^{N}=$ $\left(\mathbf{u}\left(x_{j}\right)\right)_{j=0}^{N} \in \Omega\left(\hat{\bar{\omega}}_{h}, r(\cdot)\right)$ which can be determined by the modified fixed point iteration

$$
\begin{gathered}
\mathbf{u}_{j}^{(k)}-U\left(x_{j}, x_{j-1}\right) \mathbf{u}_{j-1}^{(k)}=Y^{j}\left(x_{j}, \mathbf{u}_{j-1}^{(k-1)}\right)-U\left(x_{j}, x_{j-1}\right) \mathbf{u}_{j-1}^{(k-1)}, \quad j=1,2, \ldots, N, \\
B_{0} \mathbf{u}_{0}^{(k)}+B_{1} \mathbf{u}_{N}^{(k)}=\mathbf{d}, \quad k=1,2, \ldots, \\
\mathbf{u}_{j}^{(0)}=U\left(x_{j}, 0\right) Q^{-1} \mathbf{d}, \quad j=0,1, \ldots, N .
\end{gathered}
$$

The corresponding error estimate is

$$
\left\|\mathbf{u}^{(k)}-\mathbf{u}\right\|_{0, \infty, \hat{\bar{\omega}}_{h}} \leq \frac{q_{1}^{k}}{1-q_{1}} r(1),
$$

where $q_{1} \equiv q \exp \left[L|h| \exp \left(c_{1}|h|\right)\right]<1$. 
8 Difference schemes for BVPs

Proof. Taking into account (2.2), we apply successively the formula (3.11) and get

$$
\begin{aligned}
\mathbf{u}_{1} & =U\left(x_{1}, 0\right) \mathbf{u}_{0}+\mathbf{Y}^{1}\left(x_{1}, \mathbf{u}_{0}\right)-U\left(x_{1}, 0\right) \mathbf{u}_{0}, \\
\mathbf{u}_{2} & =U\left(x_{2}, x_{1}\right) U\left(x_{1}, 0\right) \mathbf{u}_{0}+U\left(x_{2}, x_{1}\right)\left[\mathbf{Y}^{1}\left(x_{1}, \mathbf{u}_{0}\right)-U\left(x_{1}, 0\right) \mathbf{u}_{0}\right]+\mathbf{Y}^{2}\left(x_{2}, \mathbf{u}_{1}\right)-U\left(x_{2}, x_{1}\right) \mathbf{u}_{1} \\
& =U\left(x_{2}, 0\right) \mathbf{u}_{0}+U\left(x_{2}, x_{1}\right)\left[\mathbf{Y}^{1}\left(x_{1}, \mathbf{u}_{0}\right)-U\left(x_{1}, 0\right) \mathbf{u}_{0}\right]+\mathbf{Y}^{2}\left(x_{2}, \mathbf{u}_{1}\right)-U\left(x_{2}, x_{1}\right) \mathbf{u}_{1}, \\
& \vdots \\
\mathbf{u}_{j} & =U\left(x_{j}, 0\right) \mathbf{u}_{0}+\sum_{i=1}^{j} U\left(x_{j}, x_{i}\right)\left[\mathbf{Y}^{i}\left(x_{i}, \mathbf{u}_{i-1}\right)-U\left(x_{i}, x_{i-1}\right) \mathbf{u}_{i-1}\right] .
\end{aligned}
$$

Substituting (3.19) into the boundary condition (3.12), we obtain

$$
\left[B_{0}+B_{1} U(1,0)\right] \mathbf{u}_{0}=Q \mathbf{u}_{0}=-B_{1} \sum_{i=1}^{N} U\left(1, x_{i}\right)\left[\mathbf{Y}^{i}\left(x_{i}, \mathbf{u}_{i-1}\right)-U\left(x_{i}, x_{i-1}\right) \mathbf{u}_{i-1}\right]+\mathbf{d} .
$$

Thus,

$$
\begin{aligned}
\mathbf{u}_{j}= & -U\left(x_{j}, 0\right) H \sum_{i=1}^{N} U\left(1, x_{i}\right)\left[\mathbf{Y}^{i}\left(x_{i}, \mathbf{u}_{i-1}\right)-U\left(x_{i}, x_{i-1}\right) \mathbf{u}_{i-1}\right] \\
& +\sum_{i=1}^{j} U\left(x_{j}, x_{i}\right)\left[\mathbf{Y}^{i}\left(x_{i}, \mathbf{u}_{i-1}\right)-U\left(x_{i}, x_{i-1}\right) \mathbf{u}_{i-1}\right]+U\left(x_{j}, 0\right) Q^{-1} \mathbf{d}
\end{aligned}
$$

or

$$
\mathbf{u}_{j}=\sum_{i=1}^{N} G_{h}\left(x_{j}, x_{i}\right)\left[\mathbf{Y}^{i}\left(x_{i}, \mathbf{u}_{i-1}\right)-U\left(x_{i}, x_{i-1}\right) \mathbf{u}_{i-1}\right]+\mathbf{u}^{(0)}\left(x_{j}\right),
$$

where the discrete Green's function $G_{h}(x, \xi)$ of problem (3.11)-(3.12) is the projection onto the grid $\hat{\bar{\omega}}_{h}$ of the Green's function $G(x, \xi)$ in $(2.12)$, that is,

$$
G(x, \xi)=G_{h}(x, \xi) \quad \forall x, \xi \in \hat{\bar{\omega}}_{h} .
$$

Due to

$$
\mathbf{Y}^{i}\left(x_{i}, \mathbf{u}_{i-1}\right)-U\left(x_{i}, x_{i-1}\right) \mathbf{u}_{i-1}=\int_{x_{i-1}}^{x_{i}} U\left(x_{i}, \xi\right) \mathbf{f}\left(\xi, \mathbf{Y}^{i}\left(\xi, \mathbf{u}_{i-1}\right)\right) d \xi
$$

we have

$$
\mathfrak{R}_{h}\left(x_{j},\left(\mathbf{u}_{s}\right)_{s=0}^{N}\right)=\sum_{i=1}^{N} \int_{x_{i-1}}^{x_{i}} G\left(x_{j}, \xi\right) \mathbf{f}\left(\xi, \mathbf{Y}^{i}\left(\xi, \mathbf{u}_{i-1}\right)\right) d \xi+\mathbf{u}^{(0)}\left(x_{j}\right)
$$

Next we show that the operator (3.25) transforms the set $\Omega\left(\hat{\bar{\omega}}_{h}, r(\cdot)\right)$ into itself. 
I. P. Gavrilyuk et al. 9

Let $\left(\mathbf{v}_{j}\right)_{j=0}^{N} \in \Omega\left(\hat{\bar{\omega}}_{h}, r(\cdot)\right)$, then we have (see the proof of Lemma 3.1)

$$
\begin{aligned}
\mathbf{v}(x) & =\mathbf{Y}^{j}\left(x, \mathbf{v}_{j-1}\right) \in \Omega\left(\left[x_{j-1}, x_{j}\right], r(\cdot)\right), \quad j=1,2, \ldots, N, \\
\left\|\mathfrak{R}_{h}\left(x_{j},\left(\mathbf{v}_{s}\right)_{s=0}^{N}\right)-\mathbf{u}^{(0)}\left(x_{j}\right)\right\| & \\
& \leq K\left[\exp \left[c_{1}\left(1+x_{j}\right)\right]\|H\| \sum_{i=1}^{N} \int_{x_{i-1}}^{x_{i}} \exp \left(-c_{1} \xi\right) d \xi+\exp \left(c_{1} x_{j}\right) \sum_{i=1}^{j} \int_{x_{i-1}}^{x_{i}} \exp \left(-c_{1} \xi\right) d \xi\right] \\
& \leq K\left[\exp \left(c_{1} x_{j}\right) \sum_{i=1}^{j} \exp \left(-c_{1} x_{i-1}\right) h_{i}+\exp \left[c_{1}\left(1+x_{j}\right)\right]\|H\| \sum_{i=1}^{N} \exp \left(-c_{1} x_{i-1}\right) h_{i}\right] \\
& \leq K \exp \left(c_{1} x_{j}\right)\left[x_{j}+\|H\| \exp \left(c_{1}\right)\right]=r\left(x_{j}\right), \quad j=0,1, \ldots, N .
\end{aligned}
$$

Besides, the operator $\mathfrak{R}_{h}\left(x_{j},\left(\mathbf{u}_{s}\right)_{s=0}^{N}\right)$ is a contraction on $\Omega\left(\hat{\bar{\omega}}_{h}, r(\cdot)\right)$, since due to Lemma 3.3 and the estimate

$$
\|G(x, \xi)\| \leq \begin{cases}\exp \left[c_{1}(1+x-\xi)\right]\|H\|, & 0 \leq x \leq \xi \\ \exp \left[c_{1}(x-\xi)\right]\left[1+\|H\| \exp \left(c_{1}\right)\right], & \xi \leq x \leq 1,\end{cases}
$$

which has been proved in [14], the relation (3.22) implies

$$
\begin{aligned}
\left\|\mathfrak{R}_{h}\left(x_{j},\left(\mathbf{u}_{s}\right)_{s=0}^{N}\right)-\mathfrak{R}_{h}\left(x_{j},\left(\mathbf{v}_{s}\right)_{s=0}^{N}\right)\right\|_{0, \infty, \hat{\bar{\omega}}_{h}} \\
\leq \sum_{i=1}^{N} \exp \left[c_{1}\left(x_{j}-x_{i}\right)\right]\left[1+\|H\| \exp \left(c_{1}\right)\right] L\left(x_{i}-x_{i-1}\right) \\
\quad \times \exp \left\{c_{1}\left(x_{j}-x_{i-1}\right)+L \int_{x_{i-1}}^{x_{i}} \exp \left[c_{1}\left(x_{i}-\xi\right)\right] d \xi\right\}\left\|\mathbf{u}_{j-1}-\mathbf{v}_{j-1}\right\| \\
\leq \exp \left(c_{1} x_{j}\right)\left[1+\|H\| \exp \left(c_{1}\right)\right] L \exp \left[L|h| \exp \left(c_{1}|h|\right)\right]\|\mathbf{u}-\mathbf{v}\|_{0, \infty, \hat{\omega}_{h}} \\
\leq q \exp \left[L|h| \exp \left(c_{1}|h|\right)\right]\|\mathbf{u}-\mathbf{v}\|_{0, \infty, \hat{\bar{\omega}}_{h}}=q_{1}\|\mathbf{u}-\mathbf{v}\|_{0, \infty, \hat{\bar{\omega}}_{h}} .
\end{aligned}
$$

Since (2.9) implies $q<1$, we have $q_{1}<1$ for $h_{0}$ small enough and the operator $\mathfrak{R}_{h}\left(x_{j}\right.$, $\left.\left(\mathbf{u}_{s}\right)_{s=0}^{N}\right)$ is a contraction for all $\left(\mathbf{u}_{j}\right)_{j=0}^{N},\left(\mathbf{v}_{j}\right)_{j=0}^{N} \in \Omega\left(\hat{\bar{\omega}}_{h}, r(\cdot)\right)$. Then Banach's fixed point theorem (see, e.g., [1]) says that the two-point EDS (3.11)-(3.12) has a unique solution which can be determined by the modified fixed point iteration (3.17) with the error estimate (3.18).

\section{Implementation of two-point EDS}

In order to get a constructive compact two-point difference scheme from the two-point EDS, we replace (3.11)-(3.12) by the so-called truncated difference scheme of rank $m$ $(m$-TDS):

$$
\begin{gathered}
\mathbf{y}_{j}^{(m)}=Y^{(m) j}\left(x_{j}, \mathbf{y}_{j-1}^{(m)}\right), \quad j=1,2, \ldots, N, \\
B_{0} \mathbf{y}_{0}^{(m)}+B_{1} \mathbf{y}_{N}^{(m)}=\mathbf{d},
\end{gathered}
$$


where $m$ is a positive integer, $Y^{(m) j}\left(x_{j}, \mathbf{y}_{j-1}^{(m)}\right)$ is the numerical solution of the IVP (3.2) on the interval $\left[x_{j-1}, x_{j}\right]$ which has been obtained by some one-step method of the order $m$ (e.g., by the Taylor expansion or a Runge-Kutta method):

$$
Y^{(m) j}\left(x_{j}, \mathbf{y}_{j-1}^{(m)}\right)=\mathbf{y}_{j-1}^{(m)}+h_{j} \Phi\left(x_{j-1}, \mathbf{y}_{j-1}^{(m)}, h_{j}\right)
$$

that is, it holds that

$$
\left\|\mathbf{Y}^{(m) j}\left(x_{j}, \mathbf{u}_{j-1}\right)-\mathbf{Y}^{j}\left(x_{j}, \mathbf{u}_{j-1}\right)\right\| \leq M h_{j}^{m+1},
$$

where the increment function (see, e.g., [6]) $\Phi(x, \mathbf{u}, h)$ satisfies the consistency condition

$$
\Phi(x, \mathbf{u}, 0)=\mathbf{f}(x, \mathbf{u})-A(x) \mathbf{u} .
$$

For example, in case of the Taylor expansion we have

$$
\boldsymbol{\Phi}\left(x_{j-1}, \mathbf{y}_{j-1}^{(m)}, h_{j}\right)=\mathbf{f}\left(x_{j-1}, \mathbf{y}_{j-1}^{(m)}\right)-A\left(x_{j-1}\right) \mathbf{y}_{j-1}^{(m)}+\left.\sum_{p=2}^{m} \frac{h_{j}^{p-1}}{p !} \frac{d^{p} \mathbf{Y}^{j}\left(x, \mathbf{y}_{j-1}^{(m)}\right)}{d x^{p}}\right|_{x=x_{j-1}},
$$

and in case of an explicit $s$-stage Runge-Kutta method we have

$$
\begin{aligned}
& \boldsymbol{\Phi}\left(x_{j-1}, \mathbf{y}_{j-1}^{(m)}, h_{j}\right)=b_{1} \mathbf{k}_{1}+b_{2} \mathbf{k}_{2}+\cdots+b_{s} \mathbf{k}_{s}, \\
& \mathbf{k}_{1}=\mathbf{f}\left(x_{j-1}, \mathbf{y}_{j-1}^{(m)}\right)-A\left(x_{j-1}\right) \mathbf{y}_{j-1}^{(m)}, \\
& \mathbf{k}_{2}=\mathbf{f}\left(x_{j-1}+c_{2} h_{j}, \mathbf{y}_{j-1}^{(m)}+h_{j} a_{21} \mathbf{k}_{1}\right)-A\left(x_{j-1}+c_{2} h_{j}\right)\left(\mathbf{y}_{j-1}^{(m)}+h_{j} a_{21} \mathbf{k}_{1}\right), \\
& \quad \vdots \\
& \quad \mathbf{k}_{s}=\mathbf{f}\left(x_{j-1}+c_{s} h_{j}, \mathbf{y}_{j-1}^{(m)}+h_{j}\left(a_{s 1} \mathbf{k}_{1}+a_{s 2} \mathbf{k}_{2}+\cdots+a_{s, s-1} \mathbf{k}_{s-1}\right)\right) \\
& \quad-A\left(x_{j-1}+c_{s} h_{j}\right)\left(\mathbf{y}_{j-1}^{(m)}+h_{j}\left(a_{s 1} \mathbf{k}_{1}+a_{s 2} \mathbf{k}_{2}+\cdots+a_{s, s-1} \mathbf{k}_{s-1}\right)\right),
\end{aligned}
$$

with the corresponding real parameters $c_{i}, a_{i j}, i=2,3, \ldots, s, j=1,2, \ldots, s-1, b_{i}, i=$ $1,2, \ldots, s$.

In order to prove the existence and uniqueness of a solution of the $m$-TDS (4.1)-(4.2) and to investigate its accuracy, the next assertion is needed.

Lemma 4.1. Let the method (4.3) be of the order of accuracy m. Moreover, assume that the increment function $\Phi(x, \mathbf{u}, h)$ is sufficiently smooth, the entries $a_{p s}(x)$ of the matrix $A(x)$ belong to $\mathbb{C}^{m}[0,1]$, and there exists a real number $\Delta>0$ such that $f_{p}(x, \mathbf{u}) \in \mathbb{C}^{k, m-k}([0,1] \times$ $\Omega([0,1], r(\cdot)+\Delta))$, with $k=0,1, \ldots, m-1$ and $p=1,2, \ldots, d$. Then

$$
\begin{aligned}
& \left\|U^{(1)}\left(x_{j}, x_{j-1}\right)-U\left(x_{j}, x_{j-1}\right)\right\| \leq M h_{j}^{2}, \\
& \left\|\frac{1}{h_{j}}\left[\mathbf{Y}^{(m) j}\left(x_{j}, \mathbf{v}_{j-1}\right)-U^{(1)}\left(x_{j}, x_{j-1}\right) \mathbf{v}_{j-1}\right]\right\| \leq K+M h_{j}, \\
& \left\|\frac{1}{h_{j}}\left[\mathbf{Y}^{(m) j}\left(x_{j}, \mathbf{u}_{j-1}\right)-\mathbf{Y}^{(m) j}\left(x_{j}, \mathbf{v}_{j-1}\right)-U^{(1)}\left(x_{j}, x_{j-1}\right)\left(\mathbf{u}_{j-1}-\mathbf{v}_{j-1}\right)\right]\right\| \\
& \quad \leq\left(L+M h_{j}\right)\left\|\mathbf{u}_{j-1}-\mathbf{v}_{j-1}\right\|,
\end{aligned}
$$


where $\left(\mathbf{u}_{j}\right)_{j=0}^{N},\left(\mathbf{v}_{j}\right)_{j=0}^{N} \in \Omega\left(\hat{\bar{\omega}}_{h}, r(\cdot)+\Delta\right)$. The matrix $U^{(1)}\left(x_{j}, x_{j-1}\right)$ is defined by

$$
U^{(1)}\left(x_{j}, x_{j-1}\right)=I-h_{j} A\left(x_{j-1}\right) .
$$

Proof. Inserting $x=x_{j}$ into the Taylor expansion of the function $U\left(x, x_{j-1}\right)$ at the point $x_{j-1}$ gives

$$
U\left(x_{j}, x_{j-1}\right)=U^{(1)}\left(x_{j}, x_{j-1}\right)+\int_{x_{j-1}}^{x_{j}}\left(x_{j}-t\right) \frac{d^{2} U\left(t, x_{j-1}\right)}{d t^{2}} d t .
$$

From this equation the inequality (4.8) follows immediately.

It is easy to verify the following equalities:

$$
\begin{aligned}
& \frac{1}{h_{j}}\left[\mathbf{Y}^{(m) j}\left(x_{j}, \mathbf{v}_{j-1}\right)-U^{(1)}\left(x_{j}, x_{j-1}\right) \mathbf{v}_{j-1}\right] \\
&= \boldsymbol{\Phi}\left(x_{j-1}, \mathbf{v}_{j-1}, h_{j}\right)+A\left(x_{j-1}\right) \mathbf{v}_{j-1} \\
&= \boldsymbol{\Phi}\left(x_{j-1}, \mathbf{v}_{j-1}, 0\right)+h_{j} \frac{\partial \Phi\left(x_{j-1}, \mathbf{v}_{j-1}, \bar{h}\right)}{\partial h}+A\left(x_{j-1}\right) \mathbf{v}_{j-1} \\
&= \mathbf{f}\left(x_{j-1}, \mathbf{v}_{j-1}\right)+h_{j} \frac{\partial \boldsymbol{\Phi}\left(x_{j-1}, \mathbf{v}_{j-1}, \bar{h}\right)}{\partial h}, \\
& \frac{1}{h_{j}}\left[\mathbf{Y}^{(m) j}\left(x_{j}, \mathbf{u}_{j-1}\right)-\mathbf{Y}^{(m) j}\left(x_{j}, \mathbf{v}_{j-1}\right)-U^{(1)}\left(x_{j}, x_{j-1}\right)\left(\mathbf{u}_{j-1}-\mathbf{v}_{j-1}\right)\right] \\
&=\boldsymbol{\Phi}\left(x_{j-1}, \mathbf{u}_{j-1}, h_{j}\right)-\boldsymbol{\Phi}\left(x_{j-1}, \mathbf{v}_{j-1}, h_{j}\right)+A\left(x_{j-1}\right)\left(\mathbf{u}_{j-1}-\mathbf{v}_{j-1}\right) \\
&=\mathbf{f}\left(x_{j-1}, \mathbf{u}_{j-1}\right)-\mathbf{f}\left(x_{j-1}, \mathbf{v}_{j-1}\right)+h_{j}\left[\frac{\partial \Phi\left(x_{j-1}, \mathbf{u}_{j-1}, \bar{h}\right)}{\partial h}-\frac{\partial \Phi\left(x_{j-1}, \mathbf{v}_{j-1}, \bar{h}\right)}{\partial h}\right] \\
&=\mathbf{f}\left(x_{j-1}, \mathbf{u}_{j-1}\right)-\mathbf{f}\left(x_{j-1}, \mathbf{v}_{j-1}\right) \\
& \quad+h_{j} \int_{0}^{1} \frac{\partial^{2} \boldsymbol{\Phi}\left(x_{j-1}, \theta \mathbf{u}_{j-1}+(1-\theta) \mathbf{v}_{j-1}, \bar{h}\right)}{\partial h \partial \mathbf{u}} d \theta \cdot\left(\mathbf{u}_{j-1}-\mathbf{v}_{j-1}\right),
\end{aligned}
$$

where $\bar{h} \in(0,|h|)$, which imply (4.9)-(4.10). The proof is complete.

Now, we are in the position to prove the main result of this paper.

Theorem 4.2. Let the assumptions of Theorem 2.1 and Lemma 4.1 be satisfied. Then, there exists a real number $h_{0}>0$ such that for $|h| \leq h_{0}$ the $m$-TDS (4.1)-(4.2) possesses a unique 
12 Difference schemes for BVPs

solution which can be determined by the modified fixed point iteration

$$
\begin{gathered}
\mathbf{y}_{j}^{(m, n)}-U^{(1)}\left(x_{j}, x_{j-1}\right) \mathbf{y}_{j-1}^{(m, n)}=Y^{(m) j}\left(x_{j}, \mathbf{y}_{j-1}^{(m, n)}\right)-U^{(1)}\left(x_{j}, x_{j-1}\right) \mathbf{y}_{j-1}^{(m, n-1)}, \quad j=1,2, \ldots, N, \\
B_{0} \mathbf{y}_{0}^{(m, n)}+B_{1} \mathbf{y}_{N}^{(m, n)}=\mathbf{d}, \quad n=1,2, \ldots, \\
\mathbf{y}_{j}^{(m, 0)}=\prod_{k=1}^{j} U^{(1)}\left(x_{j-k+1}, x_{j-k}\right)\left[B_{0}+B_{1} \prod_{k=1}^{N} U^{(1)}\left(x_{N-k+1}, x_{N-k}\right)\right]^{-1} \mathbf{d}, \quad j=0,1, \ldots, N .
\end{gathered}
$$

The corresponding error estimate is

$$
\left\|\mathbf{y}^{(m, n)}-\mathbf{u}\right\|_{0, \infty, \hat{\bar{\omega}}_{h}} \leq M\left(q_{2}^{n}+|h|^{m}\right),
$$

where $q_{2} \equiv q+M|h|<1$.

Proof. From (4.1) we deduce successively

$$
\begin{aligned}
\mathbf{y}_{1}^{(m)}= & U^{(1)}\left(x_{1}, x_{0}\right) \mathbf{y}_{0}^{(m)}+\mathbf{Y}^{(m) 1}\left(x_{1}, \mathbf{y}_{0}^{(m)}\right)-U^{(1)}\left(x_{1}, x_{0}\right) \mathbf{y}_{0}^{(m)}, \\
\mathbf{y}_{2}^{(m)}= & U^{(1)}\left(x_{2}, x_{1}\right) U^{(1)}\left(x_{1}, x_{0}\right) \mathbf{y}_{0}^{(m)}+U^{(1)}\left(x_{2}, x_{1}\right) \\
& \times\left[\mathbf{Y}^{(m) 1}\left(x_{1}, \mathbf{y}_{0}^{(m)}\right)-U^{(1)}\left(x_{1}, x_{0}\right) \mathbf{y}_{0}^{(m)}\right]+\mathbf{Y}^{(m) 2}\left(x_{2}, \mathbf{y}_{1}^{(m)}\right)-U^{(1)}\left(x_{2}, x_{1}\right) \mathbf{y}_{1}^{(m)}, \\
\vdots & \\
\mathbf{y}_{j}^{(m)}= & \prod_{k=1}^{j} U^{(1)}\left(x_{j-k+1}, x_{j-k}\right) \mathbf{y}_{0}^{(m)} \\
& +\sum_{i=1}^{j} \prod_{k=1}^{j-i} U^{(1)}\left(x_{j-k+1}, x_{j-k}\right)\left[\mathbf{Y}^{(m) i}\left(x_{i}, \mathbf{y}_{i-1}^{(m)}\right)-U^{(1)}\left(x_{i}, x_{i-1}\right) \mathbf{y}_{i-1}^{(m)}\right] .
\end{aligned}
$$

Substituting $\mathbf{y}_{N}^{(m)}$ into the boundary conditions (4.2), we get

$$
\begin{aligned}
{\left[B_{0}\right.} & \left.+B_{1} \prod_{k=1}^{N} U^{(1)}\left(x_{N-k+1}, x_{N-k}\right)\right] \mathbf{y}_{0}^{(m)} \\
& =-B_{1} \sum_{i=1}^{N} \prod_{k=1}^{N-i} U^{(1)}\left(x_{N-k+1}, x_{N-k}\right)\left[\mathbf{Y}^{(m) i}\left(x_{i}, \mathbf{y}_{i-1}^{(m)}\right)-U^{(1)}\left(x_{i}, x_{i-1}\right) \mathbf{y}_{i-1}^{(m)}\right]+\mathbf{d} .
\end{aligned}
$$


Let us show that the matrix in square brackets is regular. Here and in the following we use the inequality

$$
\begin{aligned}
\left\|U^{(1)}\left(x_{j}, x_{j-1}\right)\right\| & \leq\left\|U\left(x_{j}, x_{j-1}\right)\right\|+\left\|U^{(1)}\left(x_{j}, x_{j-1}\right)-U\left(x_{j}, x_{j-1}\right)\right\| \\
& \leq \exp \left(c_{1} h_{j}\right)+M h_{j}^{2}
\end{aligned}
$$

which can be easily derived using the estimate (4.8). We have

$$
\begin{aligned}
\|\left[B_{0}+\right. & \left.B_{1} \prod_{k=1}^{N} U^{(1)}\left(x_{N-k+1}, x_{N-k}\right)\right]-\left[B_{0}+B_{1} U(1,0)\right] \| \\
= & \left\|B_{1}\left[\prod_{k=1}^{N} U^{(1)}\left(x_{N-k+1}, x_{N-k}\right)-\prod_{k=1}^{N} U\left(x_{N-k+1}, x_{N-k}\right)\right]\right\| \\
= & \| B_{1} \sum_{j=1}^{N} U\left(x_{N}, x_{N-j+1}\right)\left[U^{(1)}\left(x_{N-j+1}, x_{N-j}\right)-U\left(x_{N-j+1}, x_{N-j}\right)\right] \\
& \times \prod_{i=j+1}^{N} U^{(1)}\left(x_{N-i+1}, x_{N-i}\right) \| \\
\leq & \left\|B_{1}\right\| \sum_{j=1}^{N} \exp \left[\left(1-x_{N-j+1}\right) c_{1}\right] M h_{N-j+1}^{2} \prod_{i=j+1}^{N} \exp \left[\left(c_{1} h_{N-i+1}\right)+M h_{N-i+1}^{2}\right] \\
\leq & M|h|,
\end{aligned}
$$

that is,

$$
\left\|\left[B_{0}+B_{1} \prod_{k=1}^{N} U^{(1)}\left(x_{N-k+1}, x_{N-k}\right)\right]-\left[B_{0}+B_{1} U(1,0)\right]\right\|<1
$$

for $h_{0}$ small enough. Here we have used the inequality

$$
\begin{aligned}
& \prod_{i=j+1}^{N} \exp \left[\left(c_{1} h_{N-i+1}\right)+M h_{N-i+1}^{2}\right] \\
& \quad \leq \exp \left(c_{1}\right)\left(1+M|h|^{2}\right)^{N-j} \leq \exp \left(c_{1}\right) \exp \left[M(N-j)|h|^{2}\right] \\
& \quad \leq \exp \left(c_{1}\right) \exp \left[M_{1}|h|\right] \leq \exp \left(c_{1}\right)+M|h|
\end{aligned}
$$

Since $Q=B_{0}+B_{1} U(1,0)$ is nonsingular, it follows from (4.20) that the inverse

$$
\left[B_{0}+B_{1} \prod_{k=1}^{N} U^{(m)}\left(x_{N-k+1}, x_{N-k}\right)\right]^{-1}
$$


exists and due to (4.19) the following estimate holds:

$$
\begin{gathered}
\left\|\left[B_{0}+B_{1} \prod_{k=1}^{N} U^{(1)}\left(x_{N-k+1}, x_{N-k}\right)\right]^{-1} B_{1}\right\| \\
\leq\left\|Q^{-1} B_{1}\right\|+\|\left[B_{0}+B_{1} \prod_{k=1}^{N} U^{(1)}\left(x_{N-k+1}, x_{N-k}\right)\right]^{-1} \\
-\left[B_{0}+B_{1} \prod_{k=1}^{N} U\left(x_{N-k+1}, x_{N-k}\right)\right]^{-1}\|\| B_{1} \| \\
\leq\|H\|+M|h| .
\end{gathered}
$$

Moreover, from (4.16) and (4.17) we have

$$
\begin{aligned}
\mathbf{y}_{j}^{(m)}= & -\prod_{k=1}^{j} U^{(1)}\left(x_{j-k+1}, x_{j-k}\right)\left[B_{0}+B_{1} \prod_{k=1}^{N} U^{(1)}\left(x_{N-k+1}, x_{N-k}\right)\right]^{-1} \\
& \times B_{1} \sum_{i=1}^{N} \prod_{k=1}^{N-i} U^{(1)}\left(x_{N-k+1}, x_{N-k}\right)\left[\mathbf{Y}^{(m) i}\left(x_{i}, \mathbf{y}_{i-1}^{(m)}\right)-U^{(1)}\left(x_{i}, x_{i-1}\right) \mathbf{y}_{i-1}^{(m)}\right] \\
& +\sum_{i=1}^{j} \prod_{k=1}^{j-i} U^{(1)}\left(x_{j-k+1}, x_{j-k}\right)\left[\mathbf{Y}^{(m) i}\left(x_{i}, \mathbf{y}_{i-1}^{(m)}\right)-U^{(1)}\left(x_{i}, x_{i-1}\right) \mathbf{y}_{i-1}^{(m)}\right] \\
& +\prod_{k=1}^{j} U^{(1)}\left(x_{j-k+1}, x_{j-k}\right)\left[B_{0}+B_{1} \prod_{k=1}^{N} U^{(1)}\left(x_{N-k+1}, x_{N-k}\right)\right]^{-1} \mathbf{d},
\end{aligned}
$$

or

$$
\mathbf{y}_{j}^{(m)}=\mathfrak{R}_{h}^{(m)}\left(x_{j},\left(\mathbf{y}_{s}^{(m)}\right)_{s=0}^{N}\right)
$$

where

$$
\mathfrak{R}_{h}^{(m)}\left(x_{j},\left(\mathbf{y}_{s}^{(m)}\right)_{s=0}^{N}\right)=\sum_{i=1}^{N} G_{h}^{(1)}\left(x_{j}, x_{i}\right)\left[\mathbf{Y}^{(m) i}\left(x_{i}, \mathbf{y}_{i-1}^{(m)}\right)-U^{(1)}\left(x_{i}, x_{i-1}\right) \mathbf{y}_{i-1}^{(m)}\right]+\mathbf{y}_{j}^{(m, 0)},
$$

and $G_{h}^{(1)}(x, \xi)$ is Green's function of the problem (4.1)-(4.2) given by

$$
\begin{aligned}
G_{h}^{(1)}\left(x_{j}, x_{i}\right)= & -\prod_{k=1}^{j} U^{(1)}\left(x_{j-k+1}, x_{j-k}\right)\left[B_{0}+B_{1} \prod_{k=1}^{N} U^{(1)}\left(x_{N-k+1}, x_{N-k}\right)\right]^{-1} \\
& \times B_{1} \prod_{k=1}^{N-i} U^{(1)}\left(x_{N-k+1}, x_{N-k}\right)+ \begin{cases}0, & i \geq j, \\
\prod_{k=1}^{j-i} U^{(1)}\left(x_{j-k+1}, x_{j-k}\right), & i<j .\end{cases}
\end{aligned}
$$


Estimates (4.18) and (4.23) imply

$$
\left\|G_{h}^{(1)}\left(x_{j}, x_{i}\right)\right\| \leq \begin{cases}\exp \left[c_{1}\left(1+x_{j}-x_{i}\right)\right]\|H\|+M|h|, & i \geq j \\ \exp \left[c_{1}\left(x_{j}-x_{i}\right)\right]\left[1+\|H\| \exp \left(c_{1}\right)\right]+M|h|, & i<j\end{cases}
$$

Now we use Banach's fixed point theorem. First of all we show that the operator $\mathfrak{R}_{h}^{(m)}\left(x_{j},\left(\mathbf{v}_{k}\right)_{k=0}^{N}\right)$ transforms the set $\Omega\left(\hat{\bar{\omega}}_{h}, r(x)+\Delta\right)$ into itself. Using (4.9) and (4.28) we get, for all $\left(\mathbf{v}_{k}\right)_{k=0}^{N} \in \Omega\left(\hat{\bar{\omega}}_{h}, r(\cdot)+\Delta\right)$,

$$
\begin{aligned}
&\left\|\mathfrak{R}_{h}^{(m)}\left(x_{j},\left(\mathbf{v}_{k}\right)_{k=0}^{N}\right)-\mathbf{u}^{(0)}\left(x_{j}\right)\right\| \\
& \leq(K+M|h|)\left\{\exp \left[c_{1}\left(1+x_{j}\right)\right]\|H\| \sum_{i=1}^{N} h_{i} \exp \left(-c_{1} x_{i-1}\right)\right. \\
&\left.+\exp \left(c_{1} x_{j}\right) \sum_{i=1}^{j} h_{i} \exp \left(-c_{1} x_{i-1}\right)+M|h|\right\}+M|h| \\
& \leq(K+M|h|) \exp \left(c_{1} x_{j}\right)\left[x_{j}+\|H\| \exp \left(c_{1}\right)+M|h|\right]+M|h| \\
& \leq r\left(x_{j}\right)+M|h| \leq r\left(x_{j}\right)+\Delta .
\end{aligned}
$$

It remains to show that $\mathfrak{R}_{h}^{(m)}\left(x_{j},\left(\mathbf{u}_{s}\right)_{s=0}^{N}\right)$ is a contractive operator. Due to (4.10) and (4.28) we have

$$
\begin{aligned}
\| \mathfrak{R}_{h}^{(m)} & \left(x_{j},\left(\mathbf{u}_{s}\right)_{s=0}^{N}\right)-\mathfrak{R}_{h}^{(m)}\left(x_{j},\left(\mathbf{v}_{s}\right)_{s=0}^{N}\right) \|_{0, \infty, \hat{\omega}_{h}} \\
\leq & {\left[\exp \left(c_{1}\right)\left(1+\|H\| \exp \left(c_{1}\right)\right)+M|h|\right] } \\
& \times \max _{1 \leq j \leq N}\left\|\frac{1}{h_{j}}\left[\mathbf{Y}^{(m) j}\left(x_{j}, \mathbf{u}_{j-1}\right)-\mathbf{Y}^{(m) j}\left(x_{j}, \mathbf{v}_{j-1}\right)-U^{(1)}\left(x_{j}, x_{j-1}\right)\left(\mathbf{u}_{j-1}-\mathbf{v}_{j-1}\right)\right]\right\| \\
\leq & {\left[\exp \left(c_{1}\right)\left(1+\|H\| \exp \left(c_{1}\right)\right)+M|h|\right][q+M|h|]\|\mathbf{u}-\mathbf{v}\|_{0, \infty, \hat{\bar{\omega}}_{h}} } \\
\leq & q_{2}\|\mathbf{u}-\mathbf{v}\|_{0, \infty, \hat{\bar{\omega}}_{h}},
\end{aligned}
$$

where $\left(\mathbf{u}_{k}\right)_{k=0}^{N},\left(\mathbf{v}_{k}\right)_{k=0}^{N} \in \Omega\left(\hat{\bar{\omega}}_{h}, r(\cdot)+\Delta\right)$ and $q_{2} \equiv[q+M|h|]<1$ provided that $h_{0}$ is small enough. This means that $\mathfrak{R}_{h}^{(m)}\left(x_{j},\left(\mathbf{u}_{s}\right)_{s=0}^{N}\right)$ is a contractive operator. Thus, the scheme (4.1)-(4.2) has a unique solution which can be determined by the modified fixed point iteration (4.14) with the error estimate

$$
\left\|\mathbf{y}^{(m, n)}-\mathbf{y}^{(m)}\right\|_{0, \infty, \hat{\bar{\omega}}_{h}} \leq \frac{q_{2}^{n}}{1-q_{2}}(r(1)+\Delta) .
$$


The error $\mathbf{z}_{j}^{(m)}=\mathbf{y}_{j}^{(m)}-\mathbf{u}_{j}$ of the solution of scheme (4.1)-(4.2) satisfies

$$
\begin{gathered}
\mathbf{z}_{j}^{(m)}-U^{(1)}\left(x_{j}, x_{j-1}\right) z_{j-1}^{(m)}=\psi^{(m)}\left(x_{j}, \mathbf{y}_{j-1}^{(m)}\right), \quad j=1,2, \ldots, N \\
B_{0} \mathbf{z}_{0}^{(m)}+B_{1} \mathbf{z}_{N}^{(m)}=\mathbf{0}
\end{gathered}
$$

where the residuum (the approximation error) $\psi^{(m)}\left(x_{j}, \mathbf{y}_{j-1}^{(m)}\right)$ is given by

$$
\begin{aligned}
\psi^{(m)}\left(x_{j}, \mathbf{y}_{j-1}^{(m)}\right)= & {\left[\mathbf{Y}^{(m) j}\left(x_{j}, \mathbf{u}\left(x_{j-1}\right)\right)-\mathbf{Y}^{j}\left(x_{j}, \mathbf{u}\left(x_{j-1}\right)\right)\right] } \\
& +\left[\mathbf{Y}^{(m) j}\left(x_{j}, \mathbf{y}_{j-1}^{(m)}\right)-\mathbf{Y}^{(m) j}\left(x_{j}, \mathbf{u}\left(x_{j-1}\right)\right)-U^{(1)}\left(x_{j}, x_{j-1}\right)\left(\mathbf{y}_{j-1}^{(m)}-\mathbf{u}\left(x_{j-1}\right)\right)\right] .
\end{aligned}
$$

We rewrite problem (4.32) in the equivalent form

$$
\mathbf{z}_{j}^{(m)}=\sum_{i=1}^{N} G_{h}^{(1)}\left(x_{j}, x_{i}\right) \psi^{(m)}\left(x_{i}, \mathbf{y}_{i-1}^{(m)}\right)
$$

Then (4.28) and Lemma 4.1 imply

$$
\begin{aligned}
\left\|\mathbf{z}_{j}^{(m)}\right\| \leq & {\left[\exp \left(c_{1}\right)\left(1+\|H\| \exp \left(c_{1}\right)\right)+M|h|\right] \sum_{i=1}^{N}\left\|\psi^{(m)}\left(x_{i}, \mathbf{y}_{i-1}^{(m)}\right)\right\| } \\
\leq & {\left[\exp \left(c_{1}\right)\left(1+\|H\| \exp \left(c_{1}\right)\right)+M|h|\right] } \\
& \times\left[M|h|^{m}+\sum_{i=1}^{N} h_{i}\left(L+h_{i} M\right)\left\|\mathbf{z}_{i-1}^{(m)}\right\|\right] \\
\leq & q_{2}\left\|\mathbf{z}^{(m)}\right\|_{0, \infty, \hat{\omega}_{h}}+M|h|^{m} .
\end{aligned}
$$

The last inequality yields

$$
\left\|\mathbf{z}^{(m)}\right\|_{0, \infty, \hat{\bar{\omega}}_{h}} \leq M|h|^{m}
$$

Now, from (4.31) and (4.36) we get the error estimate for the method (4.15):

$$
\left\|\mathbf{y}^{(m, n)}-\mathbf{u}\right\|_{0, \infty, \hat{\bar{\omega}}_{h}} \leq\left\|\mathbf{y}^{(m, n)}-\mathbf{y}^{(m)}\right\|_{0, \infty, \hat{\bar{\omega}}_{h}}+\left\|\mathbf{y}^{(m)}-\mathbf{u}\right\|_{0, \infty, \hat{\bar{\omega}}_{h}} \leq M\left(q_{2}^{n}+|h|^{m}\right),
$$

which completes the proof.

Remark 4.3. Using $U^{(1)}$ (see formula (4.11)) in (4.14) instead of the fundamental matrix $U$ preserves the order of accuracy but reduces the computational costs significantly. 
Above we have shown that the nonlinear system of equations which represents the TDS can be solved by the modified fixed point iteration. But actually Newton's method is used due to its higher convergence rate. The Newton method applied to the system (4.1)-(4.2) has the form

$$
\begin{gathered}
\Delta \mathbf{y}_{j}^{(m, n)}-\frac{\partial \mathbf{Y}^{(m) j}\left(x_{j}, \mathbf{y}_{j-1}^{(m, n-1)}\right)}{\partial \mathbf{u}} \Delta \mathbf{y}_{j-1}^{(m, n)}=\mathbf{Y}^{(m) j}\left(x_{j}, \mathbf{y}_{j-1}^{(m, n-1)}\right)-\mathbf{y}_{j-1}^{(m, n-1)}, \quad j=1,2, \ldots, N, \\
B_{0} \triangle \mathbf{y}_{0}^{(m, n)}+B_{1} \triangle \mathbf{y}_{N}^{(m, n)}=\mathbf{0}, \quad n=1,2, \ldots, \\
\mathbf{y}_{j}^{(m, n)}=\mathbf{y}_{j}^{(m, n-1)}+\triangle \mathbf{y}_{j}^{(m, n)}, \quad j=0,1, \ldots, N, n=1,2, \ldots,
\end{gathered}
$$

where

$$
\begin{aligned}
\frac{\partial \mathbf{Y}^{(m) j}\left(x_{j}, \mathbf{y}_{j-1}^{(m)}\right)}{\partial \mathbf{u}} & =I+h_{j} \frac{\partial \boldsymbol{\Phi}\left(x_{j-1}, \mathbf{y}_{j-1}^{(m)}, h_{j}\right)}{\partial \mathbf{u}} \\
& =I+h_{j}\left[\frac{\partial \mathbf{f}\left(x_{j-1}, \mathbf{y}_{j-1}^{(m)}\right)}{\partial \mathbf{u}}-A\left(x_{j-1}\right)\right]+O\left(h_{j}^{2}\right)
\end{aligned}
$$

and $\partial \mathbf{f}\left(x_{j-1}, \mathbf{y}_{j-1}^{(m)}\right) / \partial \mathbf{u}$ is the Jacobian of the vector-function $\mathbf{f}(x, \mathbf{u})$ at the point $\left(x_{j-1}, \mathbf{y}_{j-1}^{(m)}\right)$.

Denoting

$$
S_{j}=\frac{\partial \mathbf{Y}^{(m) j}\left(x_{j}, \mathbf{y}_{j-1}^{(m, n-1)}\right)}{\partial \mathbf{u}},
$$

the system (4.38) can be written in the following equivalent form:

$$
\left[B_{0}+B_{1} S\right] \triangle \mathbf{y}_{0}^{(m, n)}=-B_{1} \boldsymbol{\varphi}, \quad \mathbf{y}_{0}^{(m, n)}=\mathbf{y}_{0}^{(m, n-1)}+\triangle \mathbf{y}_{0}^{(m, n)}
$$

where

$$
\begin{gathered}
S=S_{N} S_{N-1} \cdots S_{1}, \quad \boldsymbol{\varphi}=\boldsymbol{\varphi}_{N}, \quad \boldsymbol{\varphi}_{0}=\mathbf{0}, \\
\boldsymbol{\varphi}_{j}=S_{j} \boldsymbol{\varphi}_{j-1}+\mathbf{Y}^{(m) j}\left(x_{j}, \mathbf{y}_{j-1}^{(m, n-1)}\right)-\mathbf{y}_{j-1}^{(m, n-1)}, \quad j=1,2, \ldots, N .
\end{gathered}
$$

After solving system (4.41) with a $(d \times d)$-matrix (this requires $\mathcal{O}(N)$ arithmetical operations since the dimension $d$ is very small in comparison with $N$ ) the solution of the system (4.38) is then computed by

$$
\begin{aligned}
\triangle \mathbf{y}_{j}^{(m, n)} & =S_{j} S_{j-1} \cdots S_{1} \triangle \mathbf{y}_{0}^{(m, n)}+\boldsymbol{\varphi}_{j} \\
\mathbf{y}_{j}^{(m, n)} & =\mathbf{y}_{j}^{(m, n-1)}+\triangle \mathbf{y}_{j}^{(m, n)}, \quad j=1,2, \ldots, N .
\end{aligned}
$$


When using Newton's method or a quasi-Newton method, the problem of choosing an appropriate start approach $\mathbf{y}_{j}^{(m, 0)}, j=1,2, \ldots, N$, arises. If the original problem contains a natural parameter and for some values of this parameter the solution is known or can be easily obtained, then one can try to continue the solution along this parameter (see, e.g., [2, pages 344-353]). Thus, let us suppose that our problem can be written in the generic form

$$
\mathbf{u}^{\prime}(x)+A(x) \mathbf{u}=\mathbf{g}(x, \mathbf{u}, \lambda), \quad x \in(0,1), \quad B_{0} \mathbf{u}(0)+B_{1} \mathbf{u}(1)=\mathbf{d}
$$

where $\lambda$ denotes the problem parameter. We assume that for each $\lambda \in\left[\lambda_{0}, \lambda_{k}\right]$ an isolated solution $\mathbf{u}(x, \lambda)$ exists and depends smoothly on $\lambda$.

If the problem does not contain a natural parameter, then we can introduce such a parameter $\lambda$ artificially by forming the homotopy function

$$
\mathbf{g}(x, \mathbf{u}, \lambda)=\lambda \mathbf{f}(x, \mathbf{u})+(1-\lambda) \mathbf{f}_{1}(x)
$$

with a given function $\mathbf{f}_{1}(x)$ such that the problem (4.46) has a unique solution.

Now, for $\lambda=0$ the problem (4.44) is reduced to the linear BVP

$$
\mathbf{u}^{\prime}(x)+A(x) \mathbf{u}=\mathbf{f}_{1}(x), \quad x \in(0,1), \quad B_{0} \mathbf{u}(0)+B_{1} \mathbf{u}(1)=\mathbf{d},
$$

while for $\lambda=1$ we obtain our original problem (1.1).

The $m$-TDS for the problem (4.44) is of the form

$$
\begin{gathered}
\mathbf{y}_{j}^{(m)}(\lambda)=Y^{(m) j}\left(x_{j}, \mathbf{y}_{j-1}^{(m)}, \lambda\right), \quad j=1,2, \ldots, N, \\
B_{0} \mathbf{y}_{0}^{(m)}(\lambda)+B_{1} \mathbf{y}_{N}^{(m)}(\lambda)=\mathbf{d} .
\end{gathered}
$$

The differentiation by $\lambda$ yields the BVP

$$
\begin{gathered}
\frac{d \mathbf{y}_{j}^{(m)}(\lambda)}{d \lambda}=\frac{\partial Y^{(m) j}\left(x_{j}, \mathbf{y}_{j-1}^{(m)}, \lambda\right)}{\partial \lambda}+\frac{\partial Y^{(m) j}\left(x_{j}, \mathbf{y}_{j-1}^{(m)}, \lambda\right)}{\partial \mathbf{u}} \frac{d \mathbf{y}_{j}^{(m)}(\lambda)}{d \lambda}, \quad j=1,2, \ldots, N, \\
B_{0} \frac{d \mathbf{y}_{0}^{(m)}(\lambda)}{d \lambda}+B_{1} \frac{d \mathbf{y}_{N}^{(m)}(\lambda)}{d \lambda}=\mathbf{0},
\end{gathered}
$$

which can be further reduced to the following system of linear algebraic equations for the unknown function $\mathbf{v}_{0}^{(m)}(\lambda)=d \mathbf{y}_{0}^{(m)}(\lambda) / d \lambda$ :

$$
\left[B_{0}+B_{1} \tilde{S}\right] \mathbf{v}_{0}^{(m)}(\lambda)=-B_{1} \tilde{\boldsymbol{\varphi}}
$$


where

$$
\begin{gathered}
\tilde{S}=\tilde{S}_{N} \tilde{S}_{N-1} \cdots \tilde{S}_{1}, \quad \tilde{S}_{j}=\frac{\partial Y^{(m) j}\left(x_{j}, \mathbf{y}_{j-1}^{(m)}, \lambda\right)}{\partial \mathbf{u}}, \quad j=1,2, \ldots, N, \\
\tilde{\boldsymbol{\varphi}}=\tilde{\boldsymbol{\varphi}}_{N}, \quad \tilde{\boldsymbol{\varphi}}_{0}=\mathbf{0}, \quad \tilde{\boldsymbol{\varphi}}_{j}=\tilde{S}_{j} \tilde{\boldsymbol{\varphi}}_{j-1}+\frac{\partial \mathbf{Y}^{(m) j}\left(x_{j}, \mathbf{y}_{j-1}^{(m)}, \lambda\right)}{\partial \lambda}, \quad j=1,2, \ldots, N .
\end{gathered}
$$

Moreover, for $\mathbf{v}_{j}^{(m)}(\lambda)=d \mathbf{y}_{j}^{(m)}(\lambda) / d \lambda$ we have the formulas

$$
\mathbf{v}_{j}^{(m)}(\lambda)=\widetilde{S}_{j} \widetilde{S}_{j-1} \cdots \widetilde{S}_{1} \mathbf{v}_{0}^{(m)}+\widetilde{\varphi}_{j}, \quad j=1,2, \ldots, N
$$

The start approach for Newton's method can now be obtained by

$$
\mathbf{y}_{j}^{(m, 0)}(\lambda+\triangle \lambda)=\mathbf{y}_{j}^{(m)}(\lambda)+\triangle \lambda \mathbf{v}_{j}^{(m)}(\lambda), \quad j=0,1, \ldots, N
$$

Example 4.4. This BVP goes back to Troesch (see, e.g., [26]) and represents a well-known test problem for numerical software (see, e.g., [5, pages 17-18]):

$$
u^{\prime \prime}=\lambda \sinh (\lambda u), \quad x \in(0,1), \quad \lambda>0, \quad u(0)=0, \quad u(1)=1
$$

We apply the truncated difference scheme of order $m$ :

$$
\begin{gathered}
\mathbf{y}_{j}^{(m)}=\mathbf{Y}^{(m) j}\left(x_{j}, \mathbf{y}_{j-1}^{(m)}\right), \quad j=1,2, \ldots, N \\
B_{0} \mathbf{y}_{0}^{(m)}+B_{1} \mathbf{y}_{N}^{(m)}=\mathbf{d},
\end{gathered}
$$

where the following Taylor series IVP-solver is used:

$$
\begin{gathered}
\mathbf{Y}^{(m) j}\left(x_{j}, \mathbf{y}_{j-1}^{(m)}\right)=\mathbf{y}_{j-1}^{(m)}+h_{j} \mathbf{F}\left(x_{j-1}, \mathbf{y}_{j-1}^{(m)}\right)+\left.\sum_{p=2}^{m} \frac{h_{j}^{p}}{p !} \frac{d^{p} \mathbf{Y}^{j}\left(x, \mathbf{y}_{j-1}^{(m)}\right)}{d x^{p}}\right|_{x=x_{j-1}}, \\
\mathbf{y}_{j}^{(m)}=\left(\begin{array}{c}
y_{1, j}^{(m)} \\
y_{2, j}^{(m)}
\end{array}\right), \quad A=\left(\begin{array}{cc}
0 & -1 \\
0 & 0
\end{array}\right), \quad B_{0}=\left(\begin{array}{ll}
1 & 0 \\
0 & 0
\end{array}\right), \quad B_{1}=\left(\begin{array}{ll}
0 & 0 \\
1 & 0
\end{array}\right), \\
\mathbf{d}=\left(\begin{array}{l}
0 \\
1
\end{array}\right), \quad \mathbf{F}(x, \mathbf{u})=-A \mathbf{u}+\mathbf{f}(x, \mathbf{u})=\left(\begin{array}{c}
-u_{2} \\
\lambda \sinh \left(\lambda u_{1}\right)
\end{array}\right) .
\end{gathered}
$$


Let us describe the algorithm for the computation of $\mathbf{Y}^{(m) j}\left(x_{j}, \mathbf{y}_{j-1}^{(m)}\right)$ in Troesch's problem which is based on the formula in (4.55). Denoting $Y_{1, p}=(1 / p !)\left(d^{p} Y_{1}^{j}\left(x, \mathbf{y}_{j-1}^{(m)}\right) /\right.$ $\left.d x^{p}\right)\left.\right|_{x=x_{j-1}}$, we get

$$
\left.\frac{1}{p !} \frac{d^{p} \mathbf{Y}^{j}\left(x, \mathbf{y}_{j-1}^{(m)}\right)}{d x^{p}}\right|_{x=x_{j-1}}=\left(\begin{array}{c}
Y_{1, p} \\
(p+1) Y_{1, p+1}
\end{array}\right)
$$

and it can be seen that in order to compute the vectors $\left.(1 / p !)\left(d^{p} \mathbf{Y}^{j}\left(x, \mathbf{y}_{j-1}^{(m)}\right) / d x^{p}\right)\right|_{x=x_{j-1}}$ it is sufficient to find $Y_{1, p}$ as the Taylor coefficients of the function $Y_{1}^{j}\left(x, \mathbf{y}_{j-1}^{(m)}\right)$ at the point $x=x_{j-1}$. This function satisfies the IVP

$$
\begin{gathered}
\frac{d^{2} Y_{1}^{j}\left(x, \mathbf{y}_{j-1}^{(m)}\right)}{d x^{2}}=\lambda \sinh \left[\lambda Y_{1}^{j}\left(x, \mathbf{y}_{j-1}^{(m)}\right)\right], \\
Y_{1}^{j}\left(x_{j-1}, \mathbf{y}_{j-1}^{(m)}\right)=y_{1, j-1}^{(m)}, \quad \frac{d Y_{1}^{j}\left(x_{j-1}, \mathbf{y}_{j-1}^{(m)}\right)}{d x}=y_{2, j-1}^{(m)} .
\end{gathered}
$$

Let

$$
\tilde{r}(x)=\sinh \left[\lambda Y_{1}^{j}\left(x, \mathbf{y}_{j-1}^{(m)}\right)\right]=\sum_{i=0}^{\infty}\left(x-x_{j-1}\right)^{i} R_{i} .
$$

Substituting this series into the differential equation (4.57), we get

$$
Y_{1, i+2}=\frac{\lambda R_{i}}{(i+1)(i+2)}
$$

Denoting $\tilde{p}(x)=\lambda Y_{1}^{j}\left(x, \mathbf{y}_{j-1}^{(m)}\right)=\sum_{i=0}^{\infty}\left(x-x_{j-1}\right)^{i} P_{i}$, we have

$$
\tilde{r}(x)=\sinh \{\tilde{p}(x)\}, \quad \tilde{s}(x)=\cosh \{\tilde{p}(x)\}=\sum_{i=0}^{\infty}\left(x-x_{j-1}\right)^{i} S_{i} .
$$

Performing the simple transformations

$$
\tilde{r}^{\prime}=\cosh \{\tilde{p}\} \tilde{p}^{\prime}=\tilde{p}^{\prime} \tilde{s}, \quad \tilde{s}^{\prime}=\sinh \{\tilde{p}\} \tilde{p}^{\prime}=\tilde{p}^{\prime} \tilde{r}
$$

and applying formula (8.20b) from [4], we arrive at the recurrence equations

$$
\begin{gathered}
R_{i}=\frac{1}{i} \sum_{k=0}^{i-1}(i-k) S_{k} P_{i-k}, \quad S_{i}=\frac{1}{i} \sum_{k=0}^{i-1}(i-k) R_{k} P_{i-k}, \quad i=1,2, \ldots, \\
P_{i}=\lambda Y_{1, i}, \quad i=2,3, \ldots
\end{gathered}
$$

The corresponding initial conditions are

$$
P_{0}=\lambda y_{1, j-1}^{(m)}, \quad P_{1}=\lambda y_{2, j-1}^{(m)}, \quad R_{0}=\sinh \left(\lambda y_{1, j-1}^{(m)}\right), \quad S_{0}=\cosh \left(\lambda y_{1, j-1}^{(m)}\right) .
$$


The Jacobian is given by

$$
\begin{aligned}
\frac{\partial \mathbf{Y}^{(m) j}\left(x_{j}, \mathbf{y}_{j-1}^{(m)}\right)}{\partial \mathbf{u}}= & I+h_{j}\left(\begin{array}{cc}
0 & 1 \\
\lambda^{2} \cosh \left(\lambda y_{1, j-1}^{(m)}\right) & 0
\end{array}\right) \\
& +\sum_{p=2}^{m} \frac{h_{j}^{p}}{p !}\left(\begin{array}{cc}
Y_{1, p, u_{1}} & Y_{1, p, u_{2}} \\
(p+1) Y_{1, p+1, u_{1}} & (p+1) Y_{1, p+1, u_{2}}
\end{array}\right),
\end{aligned}
$$

with

$$
\mathbf{u}=\left(\begin{array}{l}
u_{1} \\
u_{2}
\end{array}\right), \quad Y_{1, p, u_{l}}=\frac{\partial Y_{1, p}\left(x_{j}, \mathbf{y}_{j-1}^{(m)}\right)}{\partial u_{l}}, \quad l=1,2 .
$$

Since the functions $Y_{1, u_{l}}\left(x, \mathbf{y}_{j-1}^{(m)}\right)=\partial Y_{1}\left(x, \mathbf{y}_{j-1}^{(m)}\right) / \partial u_{l}$ satisfy the differential equations

$$
\begin{aligned}
& \frac{d^{2} Y_{1, u_{1}}}{d x^{2}}=\lambda^{2} \cosh (\tilde{p}(x))\left(1+Y_{1, u_{1}}\right), \\
& \frac{d^{2} Y_{1, u_{2}}}{d x^{2}}=\lambda^{2} \cosh (\tilde{p}(x))\left(\left(x-x_{j-1}\right)+Y_{1, u_{2}}\right),
\end{aligned}
$$

for the computation of $Y_{1, p, u_{l}}$, we get the recurrence algorithm

$$
\begin{aligned}
Y_{1, i+2, u_{1}} & =\frac{\lambda^{2}}{(i+1)(i+2)}\left[S_{i}+\sum_{k=2}^{i} Y_{1, k, u_{1}} S_{i-k}\right], \quad i=2,3, \ldots, \\
Y_{1,2, u_{1}} & =\frac{\lambda^{2} S_{0}}{2}, \quad Y_{1,3, u_{1}}=\frac{\lambda^{2} S_{1}}{6}, \\
Y_{1, i+2, u_{2}} & =\frac{\lambda^{2}}{(i+1)(i+2)}\left[S_{i-1}+\sum_{k=2}^{i} Y_{1, k, u_{2}} S_{i-k}\right], \quad i=2,3, \ldots, \\
Y_{1,2, u_{2}} & =0, \quad Y_{1,3, u_{2}}=\frac{\lambda^{2} S_{0}}{6} .
\end{aligned}
$$

For the vector $\partial \mathbf{Y}^{(m) j}\left(x_{j}, \mathbf{y}_{j-1}^{(m)}, \lambda\right) / \partial \lambda$ we have the formula

$$
\begin{aligned}
\frac{\partial \mathbf{Y}^{(m) j}\left(x_{j}, \mathbf{y}_{j-1}^{(m)}, \lambda\right)}{\partial \lambda}= & h_{j}\left(\begin{array}{c}
0 \\
\sinh \left(\lambda y_{1, j-1}^{(m)}\right)+\lambda y_{1, j-1}^{(m)} \cosh \left(\lambda y_{1, j-1}^{(m)}\right)
\end{array}\right) \\
& +\sum_{p=2}^{m} \frac{h_{j}^{p}}{p !}\left(\begin{array}{c}
Y_{1, p, \lambda} \\
(p+1) Y_{1, p+1, \lambda}
\end{array}\right),
\end{aligned}
$$


where

$$
Y_{1, p, \lambda}=\frac{\partial Y_{1, p}\left(x_{j}, \mathbf{y}_{j-1}^{(m)}, \lambda\right)}{\partial \lambda}
$$

Taking into account that $Y_{1, \lambda}^{j}\left(x_{j}, \mathbf{y}_{j-1}^{(m)}, \lambda\right)=\partial Y_{1}^{j}\left(x_{j}, \mathbf{y}_{j-1}^{(m)}, \lambda\right) / \partial \lambda$ satisfies the differential equation

$$
\frac{d^{2} Y_{1, \lambda}^{j}}{d x^{2}}=\lambda^{2} \cosh (\tilde{p}(x)) Y_{1, \lambda}^{j}+\sinh (\tilde{p}(x))+\tilde{p}(x) \cosh (\tilde{p}(x)),
$$

we obtain for $Y_{1, p, \lambda}$ the recurrence relation

$$
\begin{gathered}
Y_{1, i+2, \lambda}=\frac{1}{(i+1)(i+2)}\left[\lambda^{2} \sum_{k=2}^{i} Y_{1, k, \lambda} S_{i-k}+R_{i}+\sum_{k=0}^{i} P_{k} S_{i-k}\right], \quad i=2,3, \ldots, \\
Y_{1,2, \lambda}=\frac{R_{0}+P_{0} S_{0}}{2}, \quad Y_{1,3, \lambda}=\frac{R_{1}+P_{0} S_{1}+P_{1} S_{0}}{6} .
\end{gathered}
$$

Taking into account the behavior of the solution we choose the grid

$$
\widehat{\bar{\omega}}_{h}=\left\{x_{j}=\frac{\exp (j \alpha / N)-1}{\exp (\alpha)-1}, j=0,1,2, \ldots, N\right\}
$$

with $\alpha<0$ which becomes dense for $x \rightarrow 1$. The step sizes of this grid are given by $h_{1}=x_{1}$ and $h_{j+1}=h_{j} \exp (\alpha / N), j=1,2, \ldots, N-1$. Note that the use of the formula $h_{j}=x_{j}-x_{j-1}$, $j=1,2, \ldots, N$, for $j \rightarrow N$ and $|\alpha|$ large enough $(\alpha=-26)$ implies a large absolute roundoff error since some of $x_{j}, x_{j-1}$ lie very close together.

The a posteriori Runge estimator was used to arrive at the right boundary with a given tolerance $\varepsilon$ : the tolerance was assumed to be achieved if the following inequality is fulfilled:

$$
\max \left\{\left\|\frac{y_{N}^{(m)}-y_{2 N}^{(m)}}{\max \left(\left|y_{2 N}^{(m)}\right|, 10^{-5}\right)}\right\|_{0, \infty, \hat{\bar{\omega}}_{h}},\left\|\frac{d y_{N}^{(m)} / d x-d y_{2 N}^{(m)} / d x}{\max \left(\left|d y_{2 N}^{(m)} / d x\right|, 10^{-5}\right)}\right\|_{0, \infty, \hat{\bar{\omega}}_{h}}\right\} \leq\left(2^{m}-1\right) \varepsilon
$$

Otherwise a doubling of the number of the grid points was made. Here $y_{N}^{(m)}$ denotes the solution of the difference scheme of the order of accuracy $m$ on the grid $\left\{x_{0}, \ldots, x_{N}\right\}$, and $y_{2 N}^{(m)}$ denotes the solution of this scheme on the grid $\left\{x_{0}, \ldots, x_{2 N}\right\}$. The difference scheme 
(a system of nonlinear algebraic equations) was solved by Newton's method with the stopping criterion

$$
\max \left\{\left\|\frac{y^{(m, n)}-y^{(m, n-1)}}{\max \left(\left|y^{(m, n)}\right|, 10^{-5}\right)}\right\|_{0, \infty, \hat{\bar{\omega}}_{h}},\left\|\frac{d y^{(m, n)} / d x-d y^{(m, n-1)} / d x}{\max \left(\left|d y^{(m, n)} / d x\right|, 10^{-5}\right)}\right\|_{0, \infty, \hat{\bar{\omega}}_{h}}\right\} \leq 0.5 \varepsilon
$$

where $n=1,2, \ldots, 10$ denotes the iteration number. Setting the value of the unknown first derivative at the point $x=0$ equal to $s$ the solution of Troesch's test problem can be represented in the form (see, e.g., [22])

$$
u(x, s)=\frac{2}{\lambda} \operatorname{arcsinh}\left(\frac{s \cdot \operatorname{sn}(\lambda x, k)}{2 \cdot \operatorname{cn}(\lambda x, k)}\right), \quad k^{2}=1-\frac{s^{2}}{4},
$$

where $\operatorname{sn}(\lambda x, k), \operatorname{cn}(\lambda x, k)$ are the elliptic Jacobi functions and the parameter $s$ satisfies the equation

$$
\frac{2}{\lambda} \operatorname{arcsinh}\left(\frac{s \cdot \operatorname{sn}(\lambda, k)}{2 \cdot \operatorname{cn}(\lambda, k)}\right)=1
$$

For example, for the parameter value $\lambda=5$ one gets $s=0.457504614063 \cdot 10^{-1}$, and for $\lambda=10$ it holds that $s=0.35833778463 \cdot 10^{-3}$. Using the homotopy method (4.52) we have computed numerical solutions of Troesch's problem (4.53) for $\lambda \in[1,62]$ using a step-size $\triangle \lambda$. The numerical results for $\lambda=10,20,30,40,45,50,61$ computed with the difference scheme of the order of accuracy 7 on the grid (4.72) with $\alpha=-26$ are given in Table 4.1, where CPU* is the time needed by the processor in order to solve the sequence of Troesch problems beginning with $\lambda=1$ and using the step $\Delta \lambda$ until the value of $\lambda$ given in the table is reached. The numerical results for $\lambda=61,62$ computed with the difference scheme of the order of accuracy 10 on the grid with $\alpha=-26$ are given in Table 4.2. The real deviation from the exact solution is given by

$$
\text { Error }=\max \left\{\left\|\frac{y^{(m)}-u}{\max \left(\left|y^{(m)}\right|, 10^{-5}\right)}\right\|_{0, \infty, \hat{\bar{\omega}}_{h}},\left\|\frac{d y^{(m)} / d x-d u / d x}{\max \left(\left|d y^{(m)} / d x\right|, 10^{-5}\right)}\right\|_{0, \infty, \hat{\bar{\omega}}_{h}}\right\} .
$$

The numerical experiments were carried out with double precision in Fortran on a PC with Intel Pentium (R) $4 \mathrm{CPU} 1700 \mathrm{MHz}$ processor and a RAM of $512 \mathrm{MB}$. To calculate the Jacobi functions $\operatorname{sn}(x, k), \operatorname{cn}(x, k)$ for large $|x|$ the computer algebra tool Maple VII with Digits $=80$ was used. Then, the exact solution on the grid $\widehat{\omega}_{h}$ and an approximation for the parameter $s$, namely, $s=0.2577072228793720338185 \cdot 10^{-25}$ satisfying $|u(1, s)-1|<0.17 \cdot 10^{-10}$ and $s=0.948051891387119532089349753 \cdot 10^{-26}$ satisfying $|u(1, s)-1|<0.315 \cdot 10^{-15}$, were calculated. 
Table 4.1. Numerical results for the TDS with $m=7(\triangle \lambda=4)$.

\begin{tabular}{cccccc}
\hline$\lambda$ & $\varepsilon$ & $N$ & $u^{\prime}(0)$ & $u(1)$ & $\mathrm{CPU}^{*}(\mathrm{~s})$ \\
\hline 10 & $10^{-7}$ & 512 & $3.5833778 \cdot 10^{-4}$ & 1 & 0.02 \\
20 & $10^{-7}$ & 512 & $1.6487734 \cdot 10^{-8}$ & 1 & 0.04 \\
30 & $10^{-7}$ & 512 & $7.4861194 \cdot 10^{-13}$ & 1 & 0.07 \\
40 & $10^{-7}$ & 512 & $3.3987988 \cdot 10^{-17}$ & 1 & 0.10 \\
45 & $10^{-7}$ & 512 & $2.2902091 \cdot 10^{-19}$ & 1 & 0.11 \\
50 & $10^{-7}$ & 1024 & $1.5430022 \cdot 10^{-21}$ & 1 & 0.15 \\
61 & $10^{-7}$ & 262144 & $2.5770722 \cdot 10^{-26}$ & 1 & 6.10 \\
\hline
\end{tabular}

Table 4.2. Numerical results for the TDS with $m=10(\triangle \lambda=2)$.

\begin{tabular}{ccccc}
\hline$\lambda$ & $\varepsilon$ & $N$ & Error & $\mathrm{CPU}^{*}(\mathrm{~s})$ \\
\hline 61 & $10^{-6}$ & 65536 & $0.860 \cdot 10^{-5}$ & 3.50 \\
61 & $10^{-8}$ & 131072 & $0.319 \cdot 10^{-7}$ & 7.17 \\
62 & $10^{-6}$ & 262144 & $0.232 \cdot 10^{-5}$ & 8.01 \\
62 & $10^{-8}$ & 262144 & $0.675 \cdot 10^{-8}$ & 15.32 \\
\hline
\end{tabular}

Table 4.3. Numerical results for the code RWPM.

\begin{tabular}{ccccccc}
\hline$\lambda$ & $m$ & it & NFUN & $u^{\prime}(0)$ & $u(1)$ & CPU $(s)$ \\
\hline 10 & 11 & 9 & 12641 & $3.5833779 \cdot 10^{-4}$ & 1.0000000 & 0.01 \\
20 & 11 & 13 & 34425 & $1.6487732 \cdot 10^{-8}$ & 0.9999997 & 0.02 \\
30 & 14 & 16 & 78798 & $7.4860938 \cdot 10^{-13}$ & 1.0000008 & 0.05 \\
40 & 15 & 24 & 172505 & $3.3986834 \cdot 10^{-17}$ & 0.9999996 & 0.14 \\
45 & 12 & 31 & 530085 & $2.2900149 \cdot 10^{-19}$ & 1.0000003 & 0.30 \\
\hline
\end{tabular}

To compare the results we have solved problem (4.53) with the multiple shooting code RWPM (see, e.g., [7] or [27]). For the parameter values $\lambda=10,20,30,40$ the numerical IVP-solver used was the code RKEX78, an implementation of the Dormand-Prince embedded Runge-Kutta method 7(8), whereas for $\lambda=45$ we have used the code BGSEXP, an implementation of the well-known Bulirsch-Stoer-Gragg extrapolation method. In Table 4.3 we denote by $m$ the number of the automatically determined shooting points, NFUN is the number of ODE calls, it the number of iterations, and CPU the CPU time used. One can observe that the accuracy characteristics of our TDS method are better than that of the code RWPM. Besides, RWPM fails for values $\lambda \geq 50$. 
Example 4.5. Let us consider the BVP for a system of stiff differential equations (see [21])

$$
\begin{gathered}
u_{1}^{\prime}=\lambda \frac{\left(u_{3}-u_{1}\right) u_{1}}{u_{2}}, \quad u_{2}^{\prime}=-\lambda\left(u_{3}-u_{1}\right), \\
u_{3}^{\prime}=\frac{0.9-10^{3}\left(u_{3}-u_{5}\right)-\lambda\left(u_{3}-u_{1}\right) u_{3}}{u_{4}}, \\
u_{4}^{\prime}=\lambda\left(u_{3}-u_{1}\right), \quad u_{5}^{\prime}=-100\left(u_{5}-u_{3}\right), \quad 0<x<1, \\
u_{1}(0)=u_{2}(0)=u_{3}(0)=1, \quad u_{4}(0)=-10, \quad u_{3}(1)=u_{5}(1) .
\end{gathered}
$$

In order to solve this problem numerically we apply the TDS of the order of accuracy 6 given by

$$
\begin{gathered}
\mathbf{y}_{j}^{(6)}=\mathbf{Y}^{(6) j}\left(x_{j}, \mathbf{y}_{j-1}^{(6)}\right), \quad j=1,2, \ldots, N \\
B_{0} \mathbf{y}_{0}^{(6)}+B_{1} \mathbf{y}_{N}^{(6)}=\mathbf{d}
\end{gathered}
$$

where $\mathbf{Y}^{(6) j}\left(x_{j}, \mathbf{y}_{j-1}^{(6)}\right)$ is the numerical solution of the IVP (3.2) computed by the following Runge-Kutta method of the order 6 (see, e.g., [6]):

$$
\begin{aligned}
\mathbf{Y}^{(6) j}\left(x_{j}, \mathbf{y}_{j-1}^{(6)}\right)= & \mathbf{y}_{j-1}^{(6)}+h_{j}\left(\frac{13}{200}\left(\mathbf{k}_{1}+\mathbf{k}_{7}\right)+\frac{11}{40}\left(\mathbf{k}_{3}+\mathbf{k}_{4}\right)+\frac{4}{25}\left(\mathbf{k}_{5}+\mathbf{k}_{6}\right)\right), \\
\mathbf{k}_{1}= & \mathbf{F}\left(x_{j-1}, \mathbf{y}_{j-1}^{(6)}\right), \\
\mathbf{k}_{2}= & \mathbf{F}\left(x_{j-1}+\frac{1}{2} h_{j}, \mathbf{y}_{j-1}^{(6)}+\frac{1}{2} h_{j} \mathbf{k}_{1}\right), \\
\mathbf{k}_{3}= & \mathbf{F}\left(x_{j-1}+\frac{2}{3} h_{j}, \mathbf{y}_{j-1}^{(6)}+\frac{2}{9} h_{j} \mathbf{k}_{1}+\frac{4}{9} h_{j} \mathbf{k}_{2}\right), \\
\mathbf{k}_{4}= & \mathbf{F}\left(x_{j-1}+\frac{1}{3} h_{j}, \mathbf{y}_{j-1}^{(6)}+\frac{7}{36} h_{j} \mathbf{k}_{1}+\frac{2}{9} h_{j} \mathbf{k}_{2}-\frac{1}{12} h_{j} \mathbf{k}_{3}\right), \\
\mathbf{k}_{5}= & \mathbf{F}\left(x_{j-1}+\frac{5}{6} h_{j}, \mathbf{y}_{j-1}^{(6)}-\frac{35}{144} h_{j} \mathbf{k}_{1}-\frac{55}{36} h_{j} \mathbf{k}_{2}+\frac{35}{48} h_{j} \mathbf{k}_{3}+\frac{15}{8} h_{j} \mathbf{k}_{4}\right), \\
\mathbf{k}_{6}= & \mathbf{F}\left(x_{j-1}+\frac{1}{6} h_{j}, \mathbf{y}_{j-1}^{(6)}-\frac{1}{360} h_{j} \mathbf{k}_{1}-\frac{11}{36} h_{j} \mathbf{k}_{2}-\frac{1}{8} h_{j} \mathbf{k}_{3}+\frac{1}{2} h_{j} \mathbf{k}_{4}+\frac{1}{10} h_{j} \mathbf{k}_{5}\right), \\
\mathbf{k}_{7}= & \mathbf{F}\left(x_{j-1}+h_{j}, \mathbf{y}_{j-1}^{(6)}-\frac{41}{260} h_{j} \mathbf{k}_{1}+\frac{22}{13} h_{j} \mathbf{k}_{2}\right. \\
\quad & \left.\quad+\frac{43}{156} h_{j} \mathbf{k}_{3}-\frac{118}{39} h_{j} \mathbf{k}_{4}+\frac{32}{195} h_{j} \mathbf{k}_{5}+\frac{80}{39} h_{j} \mathbf{k}_{6}\right) .
\end{aligned}
$$

In Newton's method (4.38) the matrix $\partial \mathbf{Y}^{(6) j}\left(x_{j}, \mathbf{y}_{j-1}^{(6)}\right) / \partial \mathbf{u}$ is approximated by

$$
\frac{\partial \mathbf{Y}^{(6) j}\left(x_{j}, \mathbf{y}_{j-1}^{(6)}\right)}{\partial \mathbf{u}} \approx I+h_{j} \frac{\partial \mathbf{F}\left(x_{j-1}, \mathbf{y}_{j-1}^{(6)}\right)}{\partial \mathbf{u}}
$$


Difference schemes for BVPs

Table 4.4. Numerical results for the TDS with $m=6(\lambda=100)$.

\begin{tabular}{ccc}
\hline$\varepsilon$ & NFUN & CPU \\
\hline $10^{-4}$ & 24500 & 0.01 \\
$10^{-6}$ & 41440 & 0.02 \\
$10^{-8}$ & 77140 & 0.04 \\
\hline
\end{tabular}

Table 4.5. Numerical results for the code RWPM $(\lambda=100)$.

\begin{tabular}{ccc}
\hline$\varepsilon$ & NFUN & $\mathrm{CPU}^{+}$ \\
\hline $10^{-4}$ & 15498 & 0.02 \\
$10^{-6}$ & 31446 & 0.04 \\
$10^{-8}$ & 52374 & 0.06 \\
\hline
\end{tabular}

Numerical results on the uniform grid

$$
\bar{\omega}_{h}=\left\{x_{j}=j h, j=0,1, \ldots, N, h=\frac{1}{N}\right\}
$$

obtained by the TDS (4.79)-(4.81) are given in Table 4.4.

This problem was also solved by the code RWPM with the semi-implicit extrapolation method SIMPR as the IVP-solver within the multiple shooting method. As the start iteration we used the solution of the problem with $\lambda=0$. The numerical results are given in Table 4.5, where $\mathrm{CPU}^{+}$denotes the aggregate time of the solution of the linear problem with $\lambda=0$ and of the problem with $\lambda=100$.

Example 4.6. Let us consider the periodic BVP (see [19])

$$
\begin{gathered}
u^{\prime \prime}=-0.05 u^{\prime}-0.02 u^{2} \sin x+0.00005 \sin x \cos ^{2} x-0.05 \cos x-0.0025 \sin x, \quad x \in(0,2 \pi), \\
u(0)=u(2 \pi), \quad u^{\prime}(0)=u^{\prime}(2 \pi),
\end{gathered}
$$

which has the exact solution $u(x)=0.05 \cos x$.

Numerical results on the uniform grid

$$
\bar{\omega}_{h}=\left\{x_{j}=j h, j=0,1, \ldots, N, h=\frac{2 \pi}{N}\right\}
$$

obtained by the TDS (4.79)-(4.81) with

$$
\begin{gathered}
\mathbf{y}_{j}^{(m)}=\left(\begin{array}{c}
y_{1, j}^{(m)} \\
y_{2, j}^{(m)}
\end{array}\right), \quad B_{0}=\left(\begin{array}{ll}
1 & 0 \\
0 & 1
\end{array}\right), \quad B_{1}=\left(\begin{array}{cc}
-1 & 0 \\
0 & -1
\end{array}\right), \quad \mathbf{d}=\left(\begin{array}{l}
0 \\
0
\end{array}\right), \\
\mathbf{F}(x, \mathbf{u})=\left(\begin{array}{c}
-u_{2} \\
-0.05\left(u_{2}+\cos x\right)+\sin x\left(0.00005 \cos ^{2} x-0.02 u_{1}^{2}-0.0025\right)
\end{array}\right)
\end{gathered}
$$


Table 4.6. Numerical results for the TDS with $m=6$.

\begin{tabular}{cccc}
\hline$\varepsilon$ & $N$ & NFUN & Error \\
\hline $10^{-4}$ & 64 & 5712 & $0.453 \cdot 10^{-8}$ \\
$10^{-6}$ & 64 & 5712 & $0.453 \cdot 10^{-8}$ \\
$10^{-8}$ & 128 & 12432 & $0.267 \cdot 10^{-11}$ \\
\hline
\end{tabular}

are given in Table 4.6.

\section{Conclusions}

The main result of this paper is a new theoretical framework for the construction of difference schemes of an arbitrarily given order of accuracy for nonlinear two-point boundary value problems. The algorithmical aspects of these schemes and their implementation are only sketched and will be discussed in detail in forthcoming papers. Note that the proposed framework enables an automatic grid generation on the basis of efficient a posteriori error estimations as it is known from the numerical codes for IVPs. More precisely, Theorem 4.2 asserts that if the coefficients of the TDS are computed by two embedded Runge-Kutta methods of the orders $m$ and $m+1$, then the corresponding difference schemes for the given BVP are of the order $m$ and $m+1$, respectively. Thus, the difference between these two numerical solutions represents an a posteriori estimate of the local error of the $m$-TDS (analogously to IVP-solvers) which can be used for an automatic and local grid refinement.

\section{Acknowledgments}

The authors would like to acknowledge the support which is provided by the Deutsche Forschungsgemeinschaft (DFG). Moreover, they thank the referees for their valuable remarks which have improved the presentation.

\section{References}

[1] R. P. Agarwal, M. Meehan, and D. O’Regan, Fixed Point Theory and Applications, Cambridge Tracts in Mathematics, vol. 141, Cambridge University Press, Cambridge, 2001.

[2] U. M. Ascher, R. M. M. Mattheij, and R. D. Russell, Numerical Solution of Boundary Value Problems for Ordinary Differential Equations, Prentice Hall Series in Computational Mathematics, Prentice Hall, New Jersey, 1988.

[3] P. Deuflhard and G. Bader, Multiple shooting techniques revisited, Numerical Treatment of Inverse Problems in Differential and Integral Equations (P. Deuflhard and E. Hairer, eds.), Birkhäuser, Massachusetts, 1983, pp. 74-94.

[4] E. Hairer, S. P. Nørsett, and G. Wanner, Solving Ordinary Differential Equations. I. Nonstiff Problems, 2nd ed., Springer Series in Computational Mathematics, vol. 8, Springer, Berlin, 1993.

[5] M. Hermann (ed.), Numerische Behandlung von Differentialgleichungen. III, Friedrich-SchillerUniversität, Jena, 1985.

[6] M. Hermann, Numerik gewöhnlicher Differentialgleichungen, Oldenbourg, München, 2004.

[7] M. Hermann and D. Kaiser, RWPM: a software package of shooting methods for nonlinear twopoint boundary value problems, Applied Numerical Mathematics 13 (1993), no. 1-3, 103-108. 
[8] M. V. Kutniv, Accurate three-point difference schemes for second-order monotone ordinary differential equations and their implementation, Computational Mathematics and Mathematical Physics 40 (2000), no. 3, 368-382, translated from Zhurnal Vychislitel'noi Matematiki i Matematicheskoi Fiziki 40 (2000), no. 3, 387-401.

[9] _ Three-point difference schemes of a high order of accuracy for systems of second-order nonlinear ordinary differential equations, Computational Mathematics and Mathematical Physics 41 (2001), no. 6, 860-873, translated from Zhurnal Vychislitel'noi Matematiki i Matematicheskoi Fiziki 41 (2001), no. 6, 909-921.

[10] _ High-order accurate three-point difference schemes for systems of second-order ordinary differential equations with a monotone operator, Computational Mathematics and Mathematical Physics 42 (2002), no. 5, 754-738, translated from Zhurnal Vychislitel'noi Matematiki i Matematicheskoi Fiziki 42 (2002)), no. 5, 754-768.

[11] Three-point difference schemes of high accuracy order for systems of nonlinear second order ordinary differential equations with the boundary conditions of the third art, Visnyk Lvivskogo Universytetu, Seria Prykladna Mathematyka ta Informatyka 4 (2002), 61-66 (Ukrainian).

[12] __ Modified three-point difference schemes of high-accuracy order for second order nonlinear ordinary differential equations, Computational Methods in Applied Mathematics 3 (2003), no. 2, 287-312.

[13] M. V. Kutniv, V. L. Makarov, and A. A. Samarskiü, Accurate three-point difference schemes for second-order nonlinear ordinary differential equations and their implementation, Computational Mathematics and Mathematical Physics 39 (1999), no. 1, 40-55, translated from Zhurnal Vychislitel'noi Matematiki i Matematicheskoi Fiziki 39 (1999), no. 1, 45-60.

[14] V. L. Makarov, I. P. Gavrilyuk, M. V. Kutniv, and M. Hermann, A two-point difference scheme of an arbitrary order of accuracy for BVPs for systems of first order nonlinear ODEs, Computational Methods in Applied Mathematics 4 (2004), no. 4, 464-493.

[15] V. L. Makarov and V. V. Guminskiı̆, A three-point difference scheme of a high order of accuracy for a system of second-order ordinary differential equations (the nonselfadjoint case), Differentsial'nye Uravneniya 30 (1994), no. 3, 493-502, 550 (Russian).

[16] V. L. Makarov, I. L. Makarov, and V. G. Prikazčikov, Tochnye raznostnye shemy i shemy liubogo poriadka tochnosti dlia sistem obyknovenny differencialnych uravnenij vtorogo poriadka, Differentsial'nye Uravneniya 15 (1979), 1194-1205.

[17] V. L. Makarov and A. A. Samarskiı̌, Exact three-point difference schemes for second-order nonlinear ordinary differential equations and their realization, Doklady Akademii Nauk SSSR 312 (1990), no. $4,795-800$.

[18] _ Realization of exact three-point difference schemes for second-order ordinary differential equations with piecewise smooth coefficients, Doklady Akademii Nauk SSSR 312 (1990), no. 3, $538-543$.

[19] M. Ronto and A. M. Samoilenko, Numerical-Analytic Methods in the Theory of Boundary-Value Problems, World Scientific, New Jersey, 2000.

[20] A. A. Samarskiu and V. L. Makarov, Realization of exact three-point difference schemes for secondorder ordinary differential equations with piecewise-smooth coefficients, Differentsial'nye Uravneniya 26 (1990), no. 7, 1254-1265, 1287.

[21] M. R. Scott and H. A. Watts, A systematized collection of codes for solving two-point boundary value problems, Numerical Methods for Differential Systems (A. K. Aziz, ed.), Academic Press, New York, 1976, pp. 197-227.

[22] J. Stoer and R. Bulirsch, Introduction to Numerical Analysis, Texts in Applied Mathematics, vol. 12, Springer, New York, 2002.

[23] A. N. Tihonov and A. A. Samarskiü, Homogeneous difference schemes, Žurnal Vyčislitel' noŭ Matematiki i Matematičeskoŭ Fiziki 1 (1961), 5-63 (Russian). 
[24]_ Homogeneous difference schemes of a high order of accuracy on non-uniform nets, Žurnal Vyčislitel' noı̆ Matematiki i Matematičeskoŭ Fiziki 1 (1961), 425-440 (Russian).

[25] V. A. Trenogin, Functional Analysis, "Nauka", Moscow, 1980.

[26] B. A. Troesch, A simple approach to a sensitive two-point boundary value problem, Journal of Computational Physics 21 (1976), no. 3, 279-290.

[27] W. Wallisch and M. Hermann, Schießverfahren zur Lösung von Rand- und Eigenwertaufgaben, Teubner-Texte zur Mathematik, vol. 75, BSB B. G. Teubner, Leipzig, 1985.

I. P. Gavrilyuk: Berufsakademie Thüringen, Staatliche Studienakademie, Am Wartenberg 2, 99817 Eisenach, Germany

E-mail address: ipg@ba-eisenach.de

M. Hermann: Institute of Applied Mathematics, Friedrich Schiller University, Ernst-Abbe-Platz 1-4, 07740 Jena, Germany

E-mail address: hermann@mathematik.uni-jena.de

M. V. Kutniv: Lviv Polytechnic National University, 12 St. Bandery Street, 79013 Lviv, Ukraine E-mail address: kutniv@yahoo.com

V. L. Makarov: Department of Numerical Analysis, Institute of Mathematics, National Academy of Sciences of Ukraine, 3 Tereshchenkivs'ka Street, 01601 Kyiv-4, Ukraine

E-mail address: makarov@imath.kiev.ua 